LPTHE-05-10

SISSA-24/2005/FM

\title{
Topological Vector Symmetry of BRSTQFT and Construction of Maximal Supersymmetry
}

\author{
L. Baulieu*, G. Bossard ${ }^{\dagger}$ and A. Tanzini ${ }^{\ddagger}$ \\ ${ }^{*}$ LPTHE, CNRS and Universités Paris VI - Paris VII, Paris, France ${ }^{\S}$ \\ ${ }^{*}$ Department of Physics and Astronomy, Rutgers University ${ }^{\top}$ \\ ${ }^{\dagger}$ SISSA, Trieste, Italy $\|$
}

\begin{abstract}
The scalar and vector topological Yang-Mills symmetries determine a closed and consistent sector of Yang-Mills supersymmetry. We provide a geometrical construction of these symmetries, based on a horizontality condition on reducible manifolds. This yields globally well-defined scalar and vector topological BRST operators. These operators generate a subalgebra of maximally supersymmetric Yang-Mills theory, which is small enough to be closed off-shell with a finite set of auxiliary fields and large enough to determine the Yang-Mills supersymmetric theory. Poincaré supersymmetry is reached in the limit of flat manifolds. The arbitrariness of the gauge functions in BRSTQFTs is thus removed by the requirement of scalar and vector topological symmetry, which also determines the complete supersymmetry transformations in a twisted way. Provided additional Killing vectors exist on the manifold, an equivariant extension of our geometrical framework is provided, and the resulting "equivariant topological field theory" corresponds to the twist of super Yang-Mills theory on $\Omega$ backgrounds.
\end{abstract}

\footnotetext{
*email address: baulieu@lpthe.jussieu.fr

$\dagger$ email address: bossard@lpthe.jussieu.fr

$\ddagger$ email address: tanzini@fm.sissa.it

$\S 4$ place Jussieu, F-75252 Paris Cedex 05, France.

ฯ Piscataway, NJ08855-0849, USA.

$\|_{\text {via Beirut 2/4, 34014Trieste, Italy }}$
} 


\section{Introduction}

Topological Yang-Mills theories have been studied extensively in various dimensions some years ago $[1,2,3,5]$. They can be defined as a BRST invariant gauge-fixing of a topological invariant, and their topological observables are determined from the cohomology of the topological BRST scalar symmetry, whose geometrical interpretation is well understood.

However, a yet unsolved mystery is their relation, by a twist operation, to Poincaré supersymmetric theories, which describes particles. There is good evidence that this relation also extends to the case of topological gravity versus supergravity [6]. In fact, since topological symmetry has a clear geometrical interpretation, it has been proposed to use it to define Poincaré supersymmetry. Here we reach an understanding of the so-called vectorial topological symmetry of TQFT's, which further support this idea.

Vector symmetry was first observed as an invariance of the Chern-Simons action, gauge-fixed in the Landau gauge [4]. Its existence can be heuristically guessed from the possible conservation of the BRST antecedent of the energy momentum tensor. For a topological action that is the twist of a supersymmetric theory, its expression is identical to the symmetry that one obtains by twisting the spinorial generators of Poincaré supersymmetry, (as for the case of the scalar topological BRST operator). In fact, the twisted formulation has been used to greatly improve the study of various non-renormalization properties of $\mathcal{N}=2$ and 4 supersymmetric theories [9].

This paper focuses to the Yang-Mills case. We show that the vectorial topological symmetry can be directly introduced, geometrically, prior to the construction of the TQFT. Basically, the vector symmetry arises when one associates reparametrization symmetry and topological symmetry in a relevant way. It is important to work on manifolds that contain at least one covariantly constant vector. Eventually, the superYang-Mills theory, with Poincaré supersymmetry, is reached by untwisting the theory, in the limit of flat manifolds.

We also use the method for constructing "equivariant topological field theories", whose observables are related to the equivariant cohomology classes of the moduli space of instantons. In fact, these topological theories can be seen as the twisted versions of the Super Yang-Mills theories on the $\Omega$ background introduced in [22], that are deformed version of ordinary supersymmetric theories.

The scalar and vector invariances of TQFT's constitute a relevant subalgebra that can be closed "off-shell". Eventually, this subalgebra is sufficient to completely determine 
the full "on-shell" set of supersymmetry generators in the flat space limit. We actually show that the invariance under scalar and vector symmetry, which we will geometrically construct, is sufficient in order to fully determine the $\mathcal{N}=2$ supersymmetric action, in 8 and 4 dimensions, respectively. In the later case the supersymmetry with its 8 generators is actually determined by the construction of 5 generators, which build a closed algebra, and in the former case, 9 generators are sufficient to determine the supersymmetry with its 16 generators. The rest of the generators, (they are self-dual tensor in the twisted form), can be considered as an effective symmetry, that one gets for free as an additional symmetry of the action. They complete the scalar and vector symmetry generators into a set that can be untwisted toward Poincaré supersymmetry. They have no geometrical interpretation in our knowledge, and, moreover, the complete set of generators cannot be closed off-shell for the case of maximal supersymmetry, with 16 generators.

Determining the TQFT, and afterward the supersymmetric theory, from a symmetry principle that has a clear geometrical meaning, appears to us as a progress. Indeed, in earlier works, after having the geometrical construction of the scalar topological BRST symmetry, the determination of the action was tantamount to that of "topological gauge functions", including self-duality equations, but was not relying on a symmetry principle. Rather, one was looking for self-duality equations for the gauge fields, which one can enforce in a BRST invariant way. The lack of a complete symmetry principle was frustrating in this construction.

The way the geometrical construction for the scalar and vector BRST symmetries works is through the construction of two nilpotent graded differential operators $s$ and $\delta$ that are nilpotent and anticommute up to a Lie derivative. These operators have a transparent meaning in the fiber bundles where the Yang-Mills field and the classical gravitational field are naturally defined. At each step of our construction, the necessary requirement of global well definition can be checked.

There is eventually a duality symmetry between $s$ and $\delta$, which merely express a symmetry between topological ghosts and antighosts. This gives a better understanding of antighosts as geometric entities, instead as BRST antecedents of the Lagrange multipliers of gauge functions, as has been traditionally done, through the notion of trivial BRST quartets.

In the generic case of 8 dimensions for maximal supersymmetry, the necessity of having a manifold with a constant vector implies that its holonomy group be $G_{2}$, or a subgroup of $G_{2}$.

The formula that we will obtain are very similar in four and eight dimensions. Our 
results can be extended in lower dimensions by using dimensional reduction. Besides $G_{2^{-}}$ manifolds, cases of interest are Calabi-Yau manifolds, provided they contain a covariantly constant vector. Eventually, we express the topological actions as a $s \delta$-exact (i.e, scalar and vector BRST-exact) term, with a nice correspondence with the Chern-Simon action. In fact our formula are reminiscent of previous one found in the general context case of "balanced topological theories", [13], with $\mathcal{N}_{T} \geq 2$, but here we manage to consider the case $\mathcal{N}_{T}=1$, with a pair of "balanced operators" ( $s$ and $\delta$ ), by using a covariantly constant vector in the manifold. Then, it is quite natural to write supersymmetric actions as a $s \delta$-exact term, but the potential cannot be generally considered as a Morse function, and it allows for ghosts and antighost that different tensor structures.

The paper is organized as follows. We first give heuristic evidence that the vector symmetry is a consequence of the possible conservation of the BRST-antecedent of the energy-momentum tensor of a TQFT - in fact it is equivalent. Then we give the important result that there is a geometrical construction of the vector symmetry which is completely independent of the idea of Poincaré supersymmetry. (We include a section for ensuring global consistency of the formula). We display the invariant action under various forms, and briefly discuss the untwisting toward supersymmetry. In the last part of the paper, we show the equivariant extension of our formulation and show the relationship with twisted supersymmetry on the $\Omega$-background.

\section{Physical evidence for the existence of a vector symmetry $\delta$ in a BRSTQFT}

Let $S$ be a BRSTQFT topological action. Its Lagrangian is reparametrization invariant. In local coordinates, $\mathcal{L}_{\xi}$ represents the action of diffeomorphisms on the fields, and we can define local functionals $L_{A \mu}(x-y)$ corresponding to each field $\varphi_{A}$ of the theory as follows:

$$
\mathcal{L}_{\xi} \varphi_{A}(x)=-\int_{M} d^{n} y \xi^{\mu}(y) L_{A \mu}(y-x)
$$

We are aware that in order this operator be globally well-defined, we should add to the Lie derivative $\mathcal{L}_{\xi}$ gauge transformations that permit its covariantization. However, in this section, we only consider operators that are basically defined modulo gauge transformations, in such a way that this subtlety is not relevant. Global requirements will be fulfilled when we will construct the vector symmetry, the existence of which we heuristically justify in this section. The reparametrization invariance of $S$ implies the "off-shell" 
conservation law :

$$
\nabla^{\nu} T_{\mu \nu}(x)=\frac{1}{\sqrt{g}} \sum_{A} \int_{M} d^{n} y L_{A \mu}(x-y) \frac{\delta^{L} S}{\delta \varphi_{A}(y)}
$$

where $\delta^{L}$ denotes the left-derivative, and $T_{\mu \nu}$ is the energy momentum tensor

$$
T_{\mu \nu}=\frac{1}{e} \delta_{a b} \frac{\delta S}{\delta e_{b}^{\mu}} e_{\nu}^{a}
$$

Up to a topological term, the action $S$ is s-exact, $S=s \int_{M} \Psi$, where the topological gauge function $\Psi$ has ghost number -1 and $s$ is a topological BRST operator, which is a scalar under reparametrization. The $s$-exactness of the action implies that the energy momentum tensor is also $s$-exact. Thus :

$$
T_{\mu \nu}=s \Lambda_{\mu \nu}
$$

where $\Lambda_{\mu \nu}$ is a local functional of the fields and has ghost number -1 . The gauge function $\Psi$ is yet arbitrary. Our aim is of removing this indetermination by a symmetry principle. The later should also be a canonical property that defines a regularized action in the path integral.

We propose that this additional requirement is that the energy momentum tensor admits a conserved BRST antecedent, modulo equations of motions, consistently with Eq.(4):

$$
\nabla^{\nu} \Lambda_{\mu \nu} \approx 0
$$

As a matter of fact, this property (5) determines the theory in the Yang-Mills case, by adjusting all coefficients in the possible topological gauge functions $\Psi$, in such a way that one eventually gets the twisted Yang-Mills supersymmetric action.

Eventually, we will transform this property into a symmetry principle.

Since $\Lambda_{\mu \nu}$ is a local functional of the fields, its conservation law must take the form

$$
\nabla^{\nu} \Lambda_{\mu \nu}(x)=\frac{1}{\sqrt{g}} \sum_{A} \int_{M} d^{n} y V_{A \mu}(x-y) \frac{\delta^{L} S}{\delta \varphi_{A}(y)}
$$

where $V_{A \mu}(x-y)$ are local functionals with ghost number -1 of the fields $\varphi_{A}$. As in the case of the energy momentum tensor, where $L_{A \mu}$ determines the diffeomorphism generators, we can define from the $V_{A \mu}$ the following vector operator $[7,8]$ :

$$
\delta \varphi_{A}(x)=-\int_{M} d^{n} y \kappa^{\mu}(y) V_{A \mu}(y-x)
$$


Here $\kappa^{\mu}(y)$ is a globally well defined given vector field (which makes a distinction as compared to the ghost $\xi^{\mu}(x)$ of the diffeomorphism symmetry). Note also that this transformation is not an infinitesimal one, $\kappa$ is a finite vector field. Asking for the invariance of the action $S$ under $\delta$-transformations restricts the choice of $\kappa$. One has indeed:

$$
\delta S=-\int_{M} d^{n} x \sum_{A} \int_{M} d^{n} y \kappa^{\mu}(y) V_{A \mu}(y-x) \frac{\delta^{L} S}{\delta \varphi_{A}(x)}=\int_{M} d^{n} x \sqrt{g} \Lambda_{\mu \nu} \nabla^{\nu} \kappa^{\mu}
$$

(In the last equality we performed an integration by parts, so the necessity of global consistency must be remembered). From Eq. (8) we deduce that the $\delta$-invariance of the action only holds if $\kappa$ is covariantly constant. We stress that, $\kappa$ being a Killing vector is sufficient, since $\Lambda_{\mu \nu}$ is generally non symmetrical, see Sect.5 for more details.

The association between a global symmetry of the theory and the conservation of a current is nothing but the Noether theorem. As a matter of fact, on any given specific case, one can redefine $\Lambda_{\mu \nu}$ by the addition of a term linear in the equations of motion, in such way that its conservation law takes the simpler form

$$
\nabla^{\nu} \Lambda_{\mu \nu}^{\prime}(x)=\sum_{A} V_{A \mu}(x) \frac{\delta^{L} S}{\delta \varphi_{A}(x)}
$$

Under this form, $\Lambda_{\mu \nu}^{\prime}$ can be identified to the Noether current associated to the $\delta$ symmetry.

The statement that the conservation equation determines the complete form of the operator $\delta$ is however a non trivial one. As we will see further down, in this heuristic derivation, $\delta$ is determined modulo gauge transformations and terms linear in the equations of motion.

The understanding of the vector symmetry requires the determination of its commutation relations with the scalar BRST symmetry. We have :

$$
\begin{aligned}
\int d^{n} y L_{\mu A}(x-y) \frac{\delta S}{\delta \varphi_{A}(y)} & =\sqrt{g} \nabla^{\nu} T_{\mu \nu}(x)=s \int d^{n} y V_{\mu A}(x-y) \frac{\delta S}{\delta \varphi_{A}(y)} \\
& =\int d^{n} y\left(s V_{\mu A}(x-y) \frac{\delta S}{\delta \varphi_{A}(y)}-(-1)^{A} V_{\mu A}(x-y) s \frac{\delta S}{\delta \varphi_{A}(y)}\right) \\
& =\int d^{n} y\left(s V_{\mu A}(x-y)+\int d^{n} z V_{\mu B}(y-z) \frac{\delta s \varphi_{A}(y)}{\delta \varphi_{B}(z)}\right) \frac{\delta S}{\delta \varphi_{A}(y)}
\end{aligned}
$$


where the sum over repeated indices is assumed, as well as the fact that the functionals derivatives are taken to the left. To prove Eq. (10), one uses the following identity, which is consequence of the $s$-invariance of $S$ :

$$
\begin{aligned}
0 & =\int_{M} d^{n} z \frac{\delta}{\varphi_{A}(y)}\left(s \varphi_{B}(z) \frac{\delta S}{\delta \varphi_{B}(z)}\right) \\
& =\int_{M} d^{n} z\left(\frac{\delta s \varphi_{B}(z)}{\delta \varphi_{A}(y)} \frac{\delta S}{\delta \varphi_{B}(z)}+(-1)^{(A+1) B} s \varphi_{B}(z) \frac{\delta}{\varphi_{A}(y)} \frac{\delta S}{\delta \varphi_{B}(z)}\right) \\
& =\int_{M} d^{n} z \frac{\delta s \varphi_{B}(z)}{\delta \varphi_{A}(y)} \frac{\delta S}{\delta \varphi_{B}(z)}+(-1)^{A} s \frac{\delta S}{\delta \varphi_{A}(y)}
\end{aligned}
$$

In fact, Eq. (10) indicates that one has

$$
\{s, \delta\} \approx \mathcal{L}_{\kappa}
$$

on all fields $\varphi_{A}$, at least modulo gauge transformations and modulo terms proportional to the equations of motion.

This point is not completely obvious. Eq. (12) would be exact if the Eq. (10) would constrain the both functionals $L_{\mu A}(x-y)$ and $s V_{\mu A}(x-y)+\int d^{n} z V_{\mu B}(y-z) \frac{\delta s \varphi_{A}(y)}{\delta \varphi_{B}(z)}$ to be equal. The indetermination of this equation reverts to the determination of the solution of the equation

$$
\int d^{n} y R_{\mu A}(x-y) \frac{\delta S}{\delta \varphi_{A}(y)}=0
$$

and the equation (12) is satisfied modulo the transformations which could be generated by the functional $R_{\mu A}$. One has actually an analogous situation for the determination of the operator $\delta$ from the conservation low of $\Lambda_{\mu \nu}$.

We first observe the existence of the following solution of Eq. (13)

$$
R_{\mu A}(x-y)=\left(R_{\mu A B}(x-y)-(-1)^{A B} R_{\mu B A}(x-y)\right) \frac{\delta S}{\delta \varphi_{B}(y)}
$$

for any local functional of the fields $R_{\mu A B}$. Because of this solution the commutation relation of $s$ and $\delta$ could be true only modulo terms involving the equations of motion. One has to check whether there are other solutions of the equation (13) that cannot be written as a term linear in the equations of motion. We assume that all local functionals which are zero when the equations of motion are satisfied are linear in the equations of motion thereself via a local functional of the fields. With this assumption, if there is another solution, we can differentiate the equation (13) with respect to $\varphi_{B}(z)$, and 
obtain

$$
\left(\int d^{n} y R_{\mu A}(x-y) \frac{\delta^{2} S}{\delta \varphi_{A}(y) \delta \varphi_{B}(z)}\right)_{\mid \frac{\delta S}{\delta \varphi_{A}}=0}=0
$$

when the equations of motion are satisfied. The functional $\frac{\delta^{2} S}{\delta \varphi_{A} \delta \varphi_{B}}$ is only degenerated in theories with constraints. The solution of the equation (15) is by definition a gauge transformation. So the general solution of the equation (13) is a sum of terms linear in the equations of motion, and of local functionals which correspond to gauge transformations (or reparametrizations in gravity). Therefore $\delta$ and its commutator with $s$ are determined modulo gauge transformations and equations of motion from (6) and (10).

At this level of the discussion, one may feel frustrated by the lack of geometrical understanding of the situation, and it appears that a direct construction of $\delta$, which satisfies Eq.(12) and has ghost number -1, is needed.

Therefore we now adopt the attitude that one must reverse the point of view, and directly construct both differential operators $s$ and $\delta$, from geometrical principles. Then the determination of the action from $s$ and $\delta$ invariances will warranty the conservation law of both the energy momentum tensor and of its $s$-antecedent $\Lambda_{\mu \nu}{ }^{1}$. The determination of the superPoincaré algebra will be a corollary, using twist arguments that are allowed on the manifold that we will use.

The following sections are devoted to the geometrical construction of the symmetries in the Yang-Mills case, for the generic dimensions 4 and 8. We will also construct abinitio the differential operators $s$ and $\delta$, with an interesting irruption of antighosts on the scenery. In fact, their geometrically interpretation will arise form a duality relation between the ghosts and the antighosts, as in balanced topological field theories.

The algebra will respect by construction the closure relation Eq.(12) that is suggested by the above heuristic discussion. Eventually, we will compute the antecedent of the energy momentum tensor, and verify that it generates the $\delta$-symmetry.

\footnotetext{
${ }^{1}$ The question of anomalies of $s$ and $\delta$ invariances is of course an interesting question
} 


\section{The gravitational and Yang-Mills horizontality con- dition for the scalar and vector topological symme- tries}

\subsection{Topological symmetry and globality requirement}

The Yang-Mills topological symmetry BRST operator $s_{\text {top }}$ is defined from the equation

$$
\left(d+s_{\text {top }}\right)(A+c)+(A+c)^{2}=F+\Psi+\Phi
$$

Ref. [1] gives the interpretation of all fields in Eq.(16).

To extend this horizontality condition and eventually define the vector symmetry, we found that we must make it compatible with reparametrization invariance, and, moreover, antighost dependent. In fact, by finding the way of combining consistently topological symmetry and reparametrization invariance, we will define scalar and vector topological invariances and reparametrization symmetry, with transformation laws that are globally well-defined. To obtain global consistency, we face the not so trivial question of expressing the transformation laws of Yang-Mills connections under reparametrizations, in the base space $M$, over which one compute the path integral. The appropriate language is wellknown: it is the fiber bundle formalism. It allows one to define connections and their curvatures, and, eventually, combine Eq.(16) with reparametrization symmetry. We will show that the symmetry transformations of the fields are most easily obtained when they are lifted in the fiber bundle. Then we will give the prescription to perform the projection on the base space, which defines the fields that one can insert in a path integral. Taking equal to zero the background connection is basically the wish for the impatient reader. The latter can identify the vector ghost that expresses the reparametrization ghost in the base space with its lifted expression in the fiber bundle. (The background connections for Lorentz and Yang-Mills invariances that we will shortly introduce, $\stackrel{\circ}{A}$ and $\stackrel{\circ}{\omega}$, define the horizontal lift of the reparametrization ghost, from $\xi$ to $\xi^{h}$. Taking $\stackrel{\circ}{A}=\stackrel{\circ}{\omega}=0$ is often possible for field configurations that one encounters in quantum field theory, so one can indeed often identify $\xi$ and $\xi^{h}$ )

Using the fiber bundle language is not an unjustified excess of mathematical rigor. It allows the construction of an action that is a well-defined integral over the whole manifold, by ensuring that the Lagrangian and the symmetry transformations involve globally well defined objects. 
To define the reparametrization symmetry, one uses the notion of spin-connection, with $\omega$ as a gauge field for the Lorentz symmetry. This allows us to define the expression of combined Lorentz and reparametrization symmetry, as was done long time ago in the case of determining and classifying gravitational anomalies [10][11][12]. We will first consider the purely gravitational bundle, and then generalize it for including the topological Yang-Mills symmetry.

Eq.(16) only involves the topological ghosts. In [1] the antighosts are considered as a trivial BRST sector, which one introduces in order to do the topological gauge-fixing. At the heart of the notion of a TQFT, there is however a mapping between the ghost and antighost Hilbert spaces. The introduction of the topological vector symmetry will unexpectedly permit a transparent geometrical interpretation of the antighosts, "dual" to that of the ghosts, with some relationship to the idea of antiBRST symmetry.

\subsubsection{Pure gravitational case}

We will construct a gravitational "horizontality condition" for defining the reparametrization symmetry and the way Yang-Mills connections and their topological ghosts transform under reparametrization. We will eventually reach an algebra that is globally well defined.

To carry out this program, we define the gravitational horizontality condition on the total gravitational principal bundle $\Pi$ over the manifold $M_{n}$, ( $n$ is either 4 or 8 ), over which we will define the path integral.

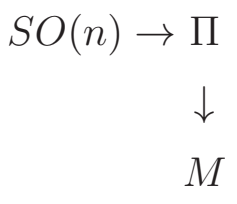

Then, we will introduce a relevant background Lorentz connection $\stackrel{\circ}{\omega}$.

A given connexion on $\Pi$ is equivalent to the selection of a decomposition of its tangent space

$$
T \Pi \cong T V \oplus T H
$$

It is known that the $\mathfrak{s o}(n)$ valued $p$-forms on $M$ are identified by the use of local trivializations to the equivariant forms in $\mathfrak{s o}(n) \otimes \Lambda^{p} T H^{*}$. The gauge potential defined on open sets of $M$ is the local trivialization of the globally well defined connection in $\mathfrak{s o}(n) \otimes T V^{*}$. In order not add too much notations, we will use the same notations for the objects defined on $\Pi$ as for their local trivializations on $M$. 
The following covariant horizontality condition on $\Pi$ defines a nilpotent and consistent graded differential operator $\mathcal{S}$, (which we donnot yet interpret), acting on the connection $\omega$ and its ghost $\Omega$

$$
(d+\mathcal{S})(\omega+\Omega)+(\omega+\Omega)^{2}=\exp \left(i_{\xi^{h}}\right) R
$$

$\xi^{h}$ is the horizontal lift on $T H_{0}$ of the reparametrization ghost vector field $\xi$ defined on $T M$. Eq. (19) is the generalization of that first found in [10] for $M$. It is aimed to determine transformations that contain local Lorentz transformations and reparametrization transformations, in the BRST formalism. The $\xi^{h}$-dependence, instead of a genuine $\xi$ dependence, involves the existence of a background connexion $\stackrel{\circ}{\omega}$, which allows us to make reparametrization explicitly compatible with (Lorentz) gauge transformations ${ }^{2} . R=d_{\omega} \omega$ is the definition of the curvature in $\Pi$.

The contraction operator $i_{\xi^{h}}$ acts on all relevant objects in $\Pi$, forms and connections. An easy computation gives the following identity on $\Pi$ :

$$
\exp \left(-i_{\xi^{h}}\right)(d+\mathcal{S}) \exp \left(i_{\xi^{h}}\right)=d+\mathcal{S}-\mathcal{L}_{\xi^{h}}+i_{\left(\mathcal{S} \xi^{h}-\frac{1}{2}\left\{\xi^{h}, \xi^{h}\right\}\right)}
$$

where $\mathcal{L}_{\xi^{h}}=\left[i_{\xi^{h}}, d\right]$.

The nilpotency of the graded operator $(d+\mathcal{S})$ amounts to that in the rhs of Eq. $(20)$. This equation implies the introduction in $M$ of a vector field $\varphi=\mathcal{S} \xi-\frac{1}{2}\{\xi, \xi\}$, that we may call a ghost of ghost of the reparametrization ghost $\xi$. We must have for consistency the following transformation laws :

$$
\begin{aligned}
& \mathcal{S} \xi=\varphi+\frac{1}{2}\{\xi, \xi\} \\
& \mathcal{S} \varphi=\mathcal{L}_{\xi} \varphi
\end{aligned}
$$

(Ref. [11] explains the algebraic details of this construction). Provided that the later equation is satisfied, nothing forbids that $\varphi \neq 0$.

$\varphi$ is a vector field on $T M$, with horizontal lift $\varphi^{h}$ on $T H_{0}$ whose possible existence is, for the moment, just a logical possibility.

In ordinary gravity, in order to interpret $\mathcal{S}$ as the BRST operator of plain reparametrization invariance, $\varphi$ must be fixed to zero. In this case, $\mathcal{S}$ just express the ordinary gravitational and Lorentz BRST symmetry. Formally, when $\varphi \neq 0$, Eq.(21) looks like the Weyl extension of the Lie algebra of diffeomorphisms.

\footnotetext{
${ }^{2}$ We note $T H_{0}$ the horizontal tangent space defined by the background connexion $\stackrel{\circ}{\omega}$.
} 
Since $\stackrel{\circ}{\omega}$ is a background field, $\mathcal{S} \stackrel{\circ}{\omega}=0$ and the BRST operator must commute with the horizontal lift that it defines, $\mathcal{S}\left(\xi^{h}\right)=(\mathcal{S} \xi)^{h}$, that is

$$
\begin{aligned}
\mathcal{S} \xi^{h} & =\varphi^{h}+\frac{1}{2}\{\xi, \xi\}^{h} \\
& =\varphi^{h}+\frac{1}{2}\left\{\xi^{h}, \xi^{h}\right\}+\left(\frac{1}{2} i_{\xi^{h}}^{2} \stackrel{\circ}{R}\right)^{v}
\end{aligned}
$$

where $\left(\frac{1}{2} i_{\xi^{h}}^{2} \stackrel{\circ}{R}\right)^{v} \equiv r^{v}$ is the fundamental vertical vector associated to the $\mathfrak{s o}(n)$-valued element $\frac{1}{2} i_{\xi^{h}}^{2} \stackrel{\circ}{R}$. Eq. (22) can be read as a definition of the background curvature $\stackrel{\circ}{R} \equiv d_{\stackrel{\circ}{\omega}} \stackrel{\circ}{\omega}$.

We can thus rewrite Eq.(20) in $\Pi$ under the following form :

$$
\exp \left(-i_{\xi^{h}}\right)(d+\mathcal{S}) \exp \left(i_{\xi^{h}}\right)=d+\mathcal{S}-\mathcal{L}_{\xi^{h}}+i_{r^{v}}+i_{\varphi^{h}}
$$

We redefine on $\Pi, \tilde{\Omega} \equiv \Omega-i_{\xi^{h}} \omega$, which must be written on $M$ as follows, by the use of local trivialization

$$
\tilde{\Omega}=\Omega-i_{\xi}(\omega-\stackrel{\circ}{\omega})
$$

Indeed $\omega-\stackrel{\circ}{\omega}$ is a tensorial form, and truly corresponds to a horizontal form on $\Pi$; we can thus finally rewrite the gravitational horizontality condition in $\Pi$ as :

$$
\left(d+\mathcal{S}-\mathcal{L}_{\xi^{h}}+i_{r^{v}}+i_{\varphi^{h}}\right)(\omega+\tilde{\Omega})+(\omega+\tilde{\Omega})^{2}=R
$$

Eq.(25) can be expanded in ghost number, and projected on $M$.

For $\varphi=0$, the resulting pure gravitational transformation laws depends on $\stackrel{\circ}{\omega}$, and are as those that were computed in [12], by asking that the gravitational BRST equations correspond to a Lie algebra.

We will actually generalize Eq.(25), when the relevant new ingredients will be introduced to combine it with the Yang-Mills topological symmetry and obtain the topological vector symmetry, with $\varphi \neq 0$. We will perform the projection at this moment. So, we momentarily leave Eq.(25) as it, and spend a few lines to comment on the operators that appear in it.

Since it is defined on $\Lambda^{\bullet} T V^{*}$, the contraction operator $\left(i_{r^{v}}\right)$ acts non trivially only on the connection. It generates a term $\frac{1}{2} i_{\xi^{h}}^{2} \stackrel{\circ}{R}$ when one expands in ghost number the horizontality condition.

$\mathcal{L}_{\xi^{h}}$ is defined as, $\mathcal{L}_{\xi^{h}} \equiv\left[i_{\xi^{h}}, d\right]$, where $d$ is the exterior derivative on $\Lambda^{\bullet} T \Pi^{*}$. It is defined for any $p$-form $w$ to be :

$$
\mathcal{L}_{\xi^{h}} w \equiv\left(\frac{d}{d t} \phi_{\xi^{h}, t}^{*} w\right)_{\mid t=0}
$$


where $\phi_{\xi^{h}, t}^{*}$ is the pullback application of the flow $\phi_{\xi^{h}, t}$, defined by

$$
\begin{aligned}
\frac{d}{d t} \phi_{\xi^{h}, t}(p) & \equiv \xi^{h} \phi_{\xi^{h}, t}(p) \\
\phi_{\xi^{h}, 0}(p) & \equiv p
\end{aligned}
$$

The curve $t \in[0,1] \rightarrow \phi_{\xi^{h}, t}(p) \in \Pi$ is the horizontal lift of $t \in[0,1] \rightarrow \phi_{\xi, t}(\pi p) \in M$ starting from $p$, where $\pi$ is the projection from $\Pi$ to $M$ of the fiber bundle. As such, $\mathcal{L}_{\xi^{h}}$ is a parallel transport generator and since the parallel transports preserves the tensoriality property of forms, $\mathcal{L}_{\xi^{h}}$ does also. It follows that the projection of $\mathcal{L}_{\xi^{h}}$ in $M$ must be locally expressed as:

$$
\mathcal{L}_{\xi}+\delta_{\text {Lorentz }}\left(i_{\xi} \stackrel{\circ}{\omega}\right)
$$

In fact, the projection in $M$ of $i_{\xi^{h}}$ is $i_{\xi}$ for a tensorial form, and $i_{\xi}(\omega-\stackrel{\circ}{\omega})$ for a connection.

We will now address the possibility $\varphi \neq 0$ by coupling gravity to Yang-Mills topological symmetry, so that $\mathcal{S}$ will have a more general interpretation, which will allow us to define the vector topological symmetry.

\subsubsection{Yang-Mills coupled to gravity}

In order to couple the Yang-Mills symmetry with gravity and obtain a horizontality condition for the topological Yang-Mills symmetry coupled to reparametrization symmetry, we introduce another (Yang-Mills) principal bundle $P$ :

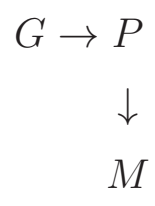

The additional horizontal Yang-Mills lift is defined by introducing a background connexion $\stackrel{\circ}{A}$ on $P$. One defines in $P$ :

$$
(d+\mathcal{S})(A+\mathcal{C})+(A+\mathcal{C})^{2}=\exp \left(i_{\xi^{h}}\right)(F+\Psi+\Phi)
$$

In an analogous way as in the previous section, we do a left-multiplication by the operator $\exp \left(-i_{\xi^{h}}\right)$, and we obtain:

$$
\left(d+\mathcal{S}-\mathcal{L}_{\xi^{h}}+i_{f^{v}}+i_{\varphi^{h}}\right)(A+\tilde{\mathcal{C}})+(A+\tilde{\mathcal{C}})^{2}=F+\Psi+\Phi
$$

where $f^{v} \equiv\left(\frac{1}{2} i_{\xi^{h}}^{2} \stackrel{\circ}{F}\right)^{v}\left(\stackrel{\circ}{F} \equiv d_{\circ} \stackrel{\circ}{A}\right)$ and $\tilde{\mathcal{C}} \equiv \mathcal{C}-i_{\xi^{h}} A$. 
To absorb the reparametrization ghost dependence, it is convenient to define a new operator $\hat{\mathcal{S}}$, from $\mathcal{S}$ [11]. For all fields, but the Faddeev-Popov ghosts $\tilde{\Omega}$ and $\tilde{\mathcal{C}}$, we define

$$
\hat{\mathcal{S}} \equiv \mathcal{S}-\mathcal{L}_{\xi}-\delta_{\text {Lorentz }}\left(i_{\xi} \stackrel{\circ}{\omega}\right)-\delta_{\text {gauge }}\left(i_{\xi} \stackrel{\circ}{A}\right)
$$

and for $\tilde{\Omega}$ and $\tilde{\mathcal{C}}$, we define :

$$
\begin{aligned}
& \hat{\mathcal{S}} \tilde{\Omega} \equiv \mathcal{S} \tilde{\Omega}-\stackrel{\circ}{\mathscr{L}}_{\xi} \tilde{\Omega}+\frac{1}{2} i_{\xi}^{2} \stackrel{\circ}{R} \\
& \hat{\mathcal{S}} \tilde{\mathcal{C}} \equiv \mathcal{S} \tilde{\mathcal{C}}-\stackrel{\circ}{\mathscr{L}}_{\xi} \tilde{\mathcal{C}}+\frac{1}{2} i_{\xi}^{2} \stackrel{\circ}{F},
\end{aligned}
$$

where $\stackrel{\circ}{\mathscr{L}}_{\xi} \equiv\left[i_{\xi}, d_{\stackrel{\circ}{\circ+\stackrel{\circ}{A}}]}\right.$.

As a consequence of $\mathcal{S}^{2}=0$, one can check that :

$$
\hat{\mathcal{S}}^{2}=-\mathcal{L}_{\varphi}-\delta_{\text {Lorentz }}\left(i_{\varphi} \stackrel{\circ}{\omega}\right)-\delta_{\text {gauge }}\left(i_{\varphi} \stackrel{\circ}{A}\right)
$$

and

$$
\hat{\mathcal{S}} d+d \hat{\mathcal{S}}=0 .
$$

Using $\hat{\mathcal{S}}$ or $\mathcal{S}$ is a matter of convenience, which depends on the problem at hand.

The "decoupled" (i.e, with no explicit $\xi$-dependence) horizontality conditions read on $\Pi$ and $P$ :

$$
\begin{aligned}
& \left(d+\hat{\mathcal{S}}+i_{\varphi^{h}}\right)(\omega+\tilde{\Omega})+(\omega+\tilde{\Omega})^{2}=R \\
& \left(d+\hat{\mathcal{S}}+i_{\varphi^{h}}\right)(A+\tilde{\mathcal{C}})+(A+\tilde{\mathcal{C}})^{2}=F+\Psi+\Phi
\end{aligned}
$$

They look almost as standard equations in flat space, except for the appearance of the operator $i_{\varphi^{h}}$.

To summarize, we started from horizontality equations that are well-defined in the fiber bundle. By projection on the manifold $M$, we obtain graded equations that determine the operator $\mathcal{S}$ in local coordinates, with transformation laws that are by construction globally well-defined, and will be shortly displayed. The redefinition of $\mathcal{S}$ into $\hat{\mathcal{S}}$ gives simple expressions.

By expansion in ghost number, the later equations (36) determines the action of the BRST operator $\hat{\mathcal{S}}$ that is equivariant with respect to the reparametrization group. After projection on $M$, they are:

$$
\begin{array}{r}
\mathcal{S} A-i_{\xi} F+d_{A} i_{\xi}(A-\stackrel{\circ}{A})+d_{A} \tilde{\mathcal{C}}=\Psi \\
\mathcal{S} \tilde{\mathcal{C}}-\stackrel{\circ}{\mathscr{L}}_{\xi} \tilde{\mathcal{C}}+\frac{1}{2}[\tilde{\mathcal{C}}, \tilde{\mathcal{C}}]+i_{\varphi}(A-\stackrel{\circ}{A})+\frac{1}{2} i_{\xi}^{2} \stackrel{\circ}{F}=\Phi
\end{array}
$$


We have similar equations for the action of $\mathcal{S}$ on $\omega$ and $\hat{\Omega}$. Using the relation between $\mathcal{S}$ and $\hat{\mathcal{S}}$, the equations can be equivalently rewritten in the following way, which shows more explicitly, term by term, that we have truly reached a globally well-defined definition of the BRST operation $\hat{\mathcal{S}}$ :

$$
\begin{aligned}
\hat{\mathcal{S}} A+d_{A} \tilde{\mathcal{C}} & =\Psi \\
\hat{\mathcal{S}} \tilde{\mathcal{C}}+\frac{1}{2}[\tilde{\mathcal{C}}, \tilde{\mathcal{C}}]+i_{\varphi}(A-\stackrel{\circ}{A}) & =\Phi
\end{aligned}
$$

This later expression of the symmetry is particularly convenient, in particular for computing the invariant Lagrangian.

\subsubsection{Putting equal to zero the background connections}

The formula are simplest when one sets to zero the background connections $\stackrel{\circ}{A}=0$ and $\stackrel{\circ}{\omega}=0$, and make no distinction between the vector fields in the fiber bundle and in $M$ (which is generally an improper choice from a global point of view, but is sufficient in perturbative quantum field theory around the trivial vacuum). This gives the transformation laws as in [11], which express the reparametrization and Yang-Mills symmetry. As indicated at the time, they express the symmetry in two equivalent ways, which are sufficient to control the symmetry of the TQFT :

$$
\begin{aligned}
\mathcal{S} A+d_{A} \mathcal{C} & =\Psi+i_{\xi} F \\
\mathcal{S C}+\frac{1}{2}[\mathcal{C}, \mathcal{C}] & =\Phi+i_{\xi} \Psi+\frac{1}{2} i_{\xi}^{2} F
\end{aligned}
$$

and

$$
\begin{aligned}
\left(\mathcal{S}-\mathcal{L}_{\xi}\right) A+d_{A} \tilde{\mathcal{C}} & =\Psi \\
\left(\mathcal{S}-\mathcal{L}_{\xi}\right) \tilde{\mathcal{C}}+\frac{1}{2}[\tilde{\mathcal{C}}, \tilde{\mathcal{C}}]+i_{\varphi} A & =\Phi
\end{aligned}
$$

where $\tilde{\mathcal{C}}=\mathcal{C}-i_{\xi} A$. The expression of $\mathcal{S}$ in Eq.(41) is explicitly covariant, but tedious to use. The expression of $\hat{\mathcal{S}} \equiv\left(\mathcal{S}-\mathcal{L}_{\xi}\right)$ in Eq.(42) is convenient, all dependence in $\xi$ is hidden, owing to the field redefinition $\mathcal{C} \rightarrow \mathcal{C}-i_{\xi} A$. Moreover, for an integral over the manifold, $\mathcal{S}$ and $\hat{\mathcal{S}}$ invariances are the same. This field redefinition looks not globally well-defined, as $i_{\xi} A$ is ambiguous from a global point of view, but the above analysis has taught us how to remedy this, it must be understood as $\mathcal{C} \rightarrow \mathcal{C}-i_{\xi}(A-\stackrel{\circ}{A})$, and for the

rest one should keep in mind the dependence in the background connection $\stackrel{\circ}{A}$, indicated in Eqs. $(32,33)$. 
We actually have a consistent recipe: global consistency is obtained from the simplest formulation with no background connection, provided one replaces all connections that may appear in the form $i_{\varphi} \omega$ or $i_{\varphi} A$ by their difference with a background connection

$i_{\varphi}(A-\stackrel{\circ}{A})$ or $i_{\varphi}(\omega-\stackrel{\circ}{\omega})$. This eventually defines a differential $\hat{\mathcal{S}}$, which encodes the relevant information on the gauge symmetry and reparametrization. $\hat{\mathcal{S}}$-invariance defines the theory.

The above presentation makes a bridge between the facts that the expression for the BRST symmetry is simplest in the fibers bundle $\Pi, P$, defined over the manifold $M$, while it needs more elaborate formula on $M$, where quantum field theory is computed. It yields unambiguously the dependence in the background connection that delivers well-defined integrals over $M$.

\subsection{Extended horizontality condition for the scalar and vector topological symmetry}

We now reach the important point of the paper, that, given a given covariantly constant vector $\kappa$ on $M$, we can geometrically build the topological vector BRST operator out of a globally well-defined operator $\hat{\mathcal{S}}$. the vector symmetry will be shortly defined as a differential graded operator $\delta$ with ghost number -1 . We understood in section 2 that a BRST-exact action can possibly define a vector symmetry that leaves it invariant. The question is to find a geometrical way of building this vector symmetry.

Since we have in mind the determination of the vector symmetry from an "extended horizontality condition", we may wish to get a hint that it is possibly contained in the geometrical formalism. We can see it from the following indirect argument, which heuristically provides evidence that the ordinary horizontality condition of a $\mathcal{N}_{T}=1 \mathrm{TQFT}$ contains the germs of another symmetry than the usual topological BRST symmetry. There are in fact topological theories with more than one scalar operator that can be identified to a BRST operator. They are known as balanced topological theories [13]. They are often obtained by dimensional reduction of a $\mathcal{N}_{T}=1$ TQFT. Such theories have a symmetry between ghosts and anti-ghost which is $S L(2, \mathbb{R})$ in the case of two charges, $\mathcal{N}_{T}=2$. Both BRST algebras can be described by a BRST-antiBRST horizontality condition, which displays a symmetry between ghosts and anti-ghosts. For instance, $\mathcal{N}_{T}=2$ occurs when one dimensionally reduces from 4 to 3 (or from 8 to 7 ) dimensions the genuine $\mathrm{d}=8$ ( or $\mathrm{d}=4) \mathcal{N}_{T}=1$ topological Yang-Mills theory. In this case, the topological ghost and antighost of the dimensionally reduced gauge field are symmetrical pair of 
anticommuting vectors that belong to the fundamental representation of $S L(2, \mathbb{R})$, and the scalar topological ghost of ghost, the corresponding antighost of antighost and the Higgs field, which results from the dimensional reduction of the Yang-Mills field, fall into the adjoint representation of $S L(2, \mathbb{R}$ ). (Of course this phenomenon is related to the property of the R-symmetry in supersymmetric theories). In these cases, dimensional reduction allows us to obtain a BRST-antiBRST symmetry from a theory that seems to have only one BRST symmetry.

Dimensional reduction occurs by giving a special role to a given dimension, and results in the elimination of the non-zero modes along a one-dimensional space $H$.

By enforcing reparametrization invariance in the relevant way, we will find, that on a reducible manifold $M \cong H \times N$ where $H \cong \mathbb{R}$ or $S_{1}$, we can construct an extended horizontality condition for $\mathcal{N}_{T}=1$ topological theories. An important point is that the invariant action will not depend on the constant vector $\kappa$ that defines the one-dimensional space $H$. This eventually defines the vector topological symmetry, which completes the ordinary scalar BRST symmetry, and shows that the $\mathcal{N}_{T}=1$ theories contain an enlarged symmetry.

For the case of $\Omega$ backgrounds, we will need the existence of a Killing vector in addition to that of a covariantly constant vector field on the manifold. Eventually, we will obtain a twisted version of a deformed supersymmetry.

In what follows, we thus consider a manifold $M$ that contains at least a constant vector. This property reduces to the reducibility property for a simply connected manifold [14]. (Reducibility only holds locally in the general case.)

\subsubsection{Obtaining of the extended horizontality condition}

We start from the formalism that introduces ghosts and antighosts of the Yang-Mills TQFT in a fully symmetrical way. We will shortly break this symmetry.

The Lorentz invariant Yang-Mills BRST-antiBRST horizontality condition is

$$
(d+s+\bar{s})(A+c+\bar{c})+(A+c+\bar{c})^{2}=F+\Psi+\bar{\Psi}+\Phi+\bar{\Phi}+L
$$

It must be completed with its Bianchi identity that determines the action of $s$ and $\bar{s}$ on the topological ghosts that occur on the right hand side, and ensures $(d+s+\bar{s})^{2}=0$. For such equations one has a conserved grading made of the sum of the ghost number and the form degree on $M$. The total ghost number of $A, c, \bar{c}, \Psi, \bar{\Psi}, \Phi, L, \bar{\Phi}$ are respectively $0,1,-1,1,-1,2,0,-2$, and their form degree $1,0,0,1,1,0,0,0$. A ghost antighost bigrading exists, such that its values for $A, c, \bar{c}, \Psi, \bar{\Psi}, \Phi, L, \bar{\Phi}$ are respectively 
$(0,0)(1,0),(0,1),(1,0),(0,1),(2,0),(1,1),(0,2)$. The scalar BRST and antiBRST operators $s$ and $\bar{s}$ have bigradings $(1,0)$ and $(0,1)$, respectively. The net ghost number of a field with ghost bigrading $\left(g, g^{\prime}\right)$ is simply $g-g^{\prime}$.

The fields $\bar{\Psi}$ and $L$ do not show up in the $\mathcal{N}_{T}=1$ theory, while the antiselfdual 2-form antighost $\chi_{2}^{-1}$ and the scalar ghost $\eta^{-1}$ of this theory does not appear in Eq.(43). We will fill this apparent contradiction and come to the point of directly determining both $s$ and $\delta$ symmetries.

We classically couple the topological theory to gravity, to express reparametrization invariance in $M$, but use the freedom of introducing a vector $\varphi \neq 0$, as generally shown in the last section. This will produces a symmetry operator $\hat{\mathcal{S}}$, which obviously contains more information than the usual scalar operator $s$.

The existence of a covariantly constant vector field $\kappa$ on the manifold basically permits one to gauge-fix the component $i_{\kappa} e^{a}$ to $\delta_{0}^{a}$. This property allows one to bypass the usual gravitational horizontality condition of the vielbeins, which imposes $\varphi$ to be null in a fully $S O(n)$ invariant theory. Some deformations of the BRST transformation of $\xi$ can in fact be consistent with the closure of $\mathcal{S}$ on $\omega$ and $\Omega$. The challenge is that the deformation must be compatible with the Bianchi identity:

$$
\mathcal{S}^{2} \omega=i_{\varphi} R \quad \mathcal{S}^{2} \Omega=i_{\xi} i_{\varphi} R
$$

Here, it is solved by some restriction of the geometry, such that the equation of motion of the first order formalism $T=0$, gives an $S O(n-1)$ holonomy curvature, leading to $i_{\kappa} R=0$. This is because the gauge fixing of the vielbeins imposes to the holonomy group of the second order curvature to be included in $S O(n-1)$.

We are thus allowed to give to $\varphi$ a non-zero value in the $\kappa$ direction. To be definite, we choose $\varphi=-\kappa$. The norm of $\kappa$ is an irrelevant quantity. Therefore, all identities must be homogeneous in $\kappa$. It is equivalent to either impose the conservation of the bigrading $\left(g, g^{\prime}\right)$, or to impose the conservation of the net ghost number $g-g^{\prime}$, assuming that $\kappa$ 's bigrading is $(1,1)$. We conjecture that this bigrading can be identified to the ghost antighost bigrading, in such a way that $g$ and $g^{\prime}$ are both positive.

We identify $\hat{\mathcal{S}}=s^{(1,0)}+s^{(0,1)}$, and $\tilde{\mathcal{C}}=c^{(1,0)}+c^{(0,1)}$. The consistent horizontality equation (36) can be written as follows:

$$
\begin{gathered}
\left(d+s^{(1,0)}+s^{(0,1)}-i_{\kappa^{h}}\right)\left(A+c^{(1,0)}+c^{(0,1)}\right)+\left(A+c^{(1,0)}+c^{(0,1)}\right)^{2} \\
=F+\Psi^{(1,0)}+\Psi^{(0,1)}+\Phi^{(2,0)}+\Phi^{(1,1)}+\Phi^{(0,2)}
\end{gathered}
$$

We now break the symmetry between the ghost and antighost sectors, using the vector field $\kappa$. Each field of $\left(g, g^{\prime}\right)$ graduation must be homogeneous of degree $g^{\prime}$ in $\kappa$, and thus 
we define:

$$
\begin{gathered}
c^{(0,1)} \equiv|\kappa| \bar{c} \quad \Psi^{(0,1)} \equiv g(\kappa) \eta+i_{\kappa} \chi \\
\Phi^{(0,2)} \equiv|\kappa|^{2} \bar{\Phi}
\end{gathered}
$$

where we defined the following 1-form out of $\kappa$ :

$$
g(\kappa) \equiv g_{\mu \nu} \kappa^{\mu} d x^{\nu}
$$

The 1-form $g(\kappa)$ satisfies $i_{\kappa} g(\kappa)=|\kappa|^{2}$, and will play an important role, together with the property $\left(i_{\kappa}\right)^{2}=0$. A non zero value of $\Phi^{(1,1)}$ defines a consistent algebra; however there is no corresponding invariant action. Therefore, we set $\Phi^{(1,1)}=0$.

Of course, $c$ and $\bar{c}$ are identified as the Faddeev-Popov ghost and antighost, respectively.

The redefinition $\Psi^{(0,1)} \rightarrow(\eta, \chi)$ is $\kappa$ dependent in a non-trivial way. In fact, the decomposition of $\Psi^{(0,1)}$ implies that the 2-form representation of the holonomy group be reducible, in order that the pair $\eta^{-1}, \chi_{\mu \nu}^{-1}$ has as many degrees of freedom as the vector $\Psi_{\mu}^{(0,1)}$. In 8 dimensions, we thus suppose that $M$ has a holonomy group not larger than $\operatorname{Spin}(7)$, so that $\chi$ be antiselfdual in the octonionic point of view in eight dimensions, with seven independent components (or $\chi$ is antiselfdual in 4 dimensions, with three independent components). Moreover, in order that $\kappa$ be globally well defined in 8 dimensions, the holonomy group must be included in $G_{2}$ (i.e, $N$ be a $G_{2}$-manifold in the reducible case).

We call:

$$
\begin{aligned}
& s=s^{(1,0)} \\
& \delta=s^{(0,1)}
\end{aligned}
$$

Having introduced all these fields, we obtain ${ }^{3}$

$$
\begin{aligned}
\left(d+s+\delta-i_{\kappa^{h}}\right)(A+c+|\kappa| \bar{c}) & +(A+c+|\kappa| \bar{c})^{2} \\
& =F+\Psi+g(\kappa) \eta+i_{\kappa^{h}} \chi+\Phi+|\kappa|^{2} \bar{\Phi}
\end{aligned}
$$

and the associated Bianchi relation

$$
\begin{aligned}
& \left(d+s+\delta-i_{\kappa^{h}}\right)\left(F+\Psi+g(\kappa) \eta+i_{\kappa^{h}} \chi+\Phi+|\kappa|^{2} \bar{\Phi}\right) \\
& +\left[A+c+|\kappa| \bar{c}, F+\Psi+g(\kappa) \eta+i_{\kappa^{h}} \chi+\Phi+|\kappa|^{2} \bar{\Phi}\right]=0
\end{aligned}
$$

\footnotetext{
${ }^{3} g(\kappa)$ must be seen on $P$ as $\pi^{*} g(\kappa)$, where $\pi$ is the projection of the fiber bundle.
} 
The property $\left(d+s+\delta-i_{\kappa^{h}}\right)^{2}=0$ implies in $M$ :

$$
\hat{\mathcal{S}}^{2}=s^{2}+\{s, \delta\}+\delta^{2}=\mathcal{L}_{\kappa}+\delta_{\text {gauge }}\left(i_{\kappa} \stackrel{\circ}{A}\right)
$$

We have therefore on all fields

$$
\begin{aligned}
s^{2} & =0 \\
\{s, \delta\} & =\mathcal{L}_{\kappa}+\delta_{\text {gauge }}\left(i_{\kappa} \stackrel{\circ}{A}\right) \\
\delta^{2} & =0
\end{aligned}
$$

And the decomposition in power of $\kappa$ of the transformation of the reparametrization ghost implies

$$
\begin{array}{ll}
s \xi=-\frac{1}{2}\{\xi, \xi\} & \delta \xi=-\kappa \\
s \kappa=0 & \delta \kappa=0
\end{array}
$$

We will see shortly that a complete and finite field representation of the algebra can be found in a consistent way in four and eight dimensions.

\subsubsection{Resolution of the extended horizontality condition}

The decomposition of the first equation (3.2.1) according to the gradings gives:

$$
\begin{array}{cc}
s A+d_{A} c=\Psi & \delta A+d_{A}|\kappa| \bar{c}=g(\kappa) \eta+i_{\kappa} \chi \\
s c+c^{2}=\Phi & \delta \bar{c}+|\kappa| \bar{c}^{2}=|\kappa| \bar{\Phi} \\
s|\kappa| \bar{c}+\delta c-i_{\kappa}(A-\stackrel{\circ}{A})+[c,|\kappa| \bar{c}]=0
\end{array}
$$

It is most convenient to use the $s$ and $\delta$ operators in the Cartan representation defined by

$$
s_{c} \equiv s+\delta_{\text {gauge }}(c) \quad \delta_{\bar{c}} \equiv \delta+\delta_{\text {gauge }}(|\kappa| \bar{c})
$$

On all fields, but $c$ and $\bar{c}$, one has:

$$
\begin{gathered}
s_{c}^{2}=\delta_{\text {gauge }}(\Phi) \quad \delta_{\bar{c}}^{2}=\delta_{\text {gauge }}\left(|\kappa|^{2} \bar{\Phi}\right) \\
\left\{s_{c}, \delta_{\bar{c}}\right\}=\mathcal{L}_{\kappa}+\delta_{\text {gauge }}\left(i_{\kappa} A\right)
\end{gathered}
$$

So, the decomposition of the Bianchi identity (3.2.1) gives

$$
\begin{array}{rr}
s_{c} \Psi+d_{A} \Phi=0 & \delta_{\bar{c}}\left(g(\kappa) \eta+i_{\kappa} \chi\right)+|\kappa|^{2} d_{A} \bar{\Phi}=0 \\
s_{c}\left(g(\kappa) \eta+i_{\kappa} \chi\right)+\delta_{\bar{c}} \Psi-i_{\kappa} F=0 \\
s_{c} \Phi=0 & \delta_{\bar{c}}|\kappa|^{2} \bar{\Phi}=0 \\
\delta_{\bar{c}} \Phi-i_{\kappa} \Psi=0 & s_{c}|\kappa|^{2} \bar{\Phi}+|\kappa|^{2} \eta=0
\end{array}
$$


Because of the bigraduation, the horizontality condition does not fully determine the action of the $s$ and $\delta$ on all fields. Indeed, one has degenerate equations of the type $s($ antighost $)+\delta$ (ghost $)=\ldots$. To raise the indetermination, we introduce "auxiliary" fields, a scalar $b^{0}$ and a 2 -form $T_{2}^{0}$, with :

$$
\begin{array}{rr}
s_{c} \chi & =T \quad s_{c} T=[\Phi, \chi] \\
s_{c} \bar{c}=b & s_{c} b=[\Phi, \bar{c}]
\end{array}
$$

It is by construction that we can consistently define the action of $s$ and $\delta$ on the "auxiliary" fields. In practice, this require a step by step computation, which yields the action of $\delta$, such that $\delta^{2}=0,\{s, \delta\}=\mathscr{L}_{\kappa}$.

The field $\chi$ occurs in the horizontality relation only trough its contraction along $\kappa$, $i_{\kappa} \chi$. Since $i_{\kappa}{ }^{2}=0$, the resolution of equation is not yet established, since $\chi$ is defined modulo terms that are $i_{\kappa}$-exact.

A little of algebra must now be done to obtain the transformation of $\chi_{\mu \nu}$. If we use the decomposition $\delta_{\bar{c}}=\kappa^{\mu} s_{\mu}$, (which is only true locally), we have from (54) and (55) that

$$
s_{[\mu} A_{\nu]}=-\chi_{\mu \nu} \quad s_{\{\mu} s_{\nu\}} A_{\sigma}=-g_{\mu \nu} D_{\sigma} \bar{\Phi}
$$

This gives

$$
s_{\sigma} \chi_{\mu \nu}=-s_{\sigma} s_{[\mu} A_{\nu]}
$$

and we deduce from the decomposition

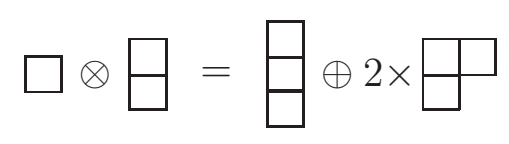

that

$$
s_{\sigma} \chi_{\mu \nu}=2 g_{\sigma[\mu} D_{\nu]} \bar{\Phi}-s_{[\sigma} s_{\mu} A_{\nu]}
$$

The ghost number and dimension of fields are such that, without introducing other fields, $s \chi$ must be proportional to $d_{A} \bar{\Phi}$

$$
s_{\sigma} \chi_{\mu \nu}=2 g_{\sigma[\mu} D_{\nu]} \bar{\Phi}-C_{[\sigma \mu \nu]}^{\star} D_{\rho} \bar{\Phi}
$$

We have introduced an invariant tensor $C_{4}^{\star}$ on the manifold. (It is the $\epsilon$ tensor for 4-manifolds, and the octonionic self-dual 4-form for Joyce manifolds.) 
We already know that the condition $\chi_{\mu \nu}=P_{\mu \nu}^{-\sigma \rho} \chi_{\sigma \rho}$, for this field to count for $n-1$ degrees of freedom in $n$ dimensions. Since $\delta_{\bar{c}}^{2} \chi=[\bar{\Phi}, \chi], C_{\mu \nu \sigma \rho}^{\star}$ must be totally antisymmetric and that

$$
\left(2 \delta_{\mu \nu}^{\{\sigma \mid \kappa}-C_{\mu \nu}^{\star}\{\sigma \mid \kappa) P^{-\rho\}}{ }_{\kappa}^{\theta \tau}=g^{\sigma \rho} P_{\mu \nu}^{-\theta \tau}\right.
$$

which gives

$$
P_{\mu \nu}^{-\sigma \rho}=\frac{2}{n}\left(\delta_{\mu \nu}^{\sigma \rho}-\frac{1}{2} C_{\mu \nu}^{\star} \sigma \rho\right)
$$

We know from [15] that the only possibilities to construct such projector with the holonomy group reduced to at most the maximal invariant subgroup of $S O(n)$ are in four and eight dimensions. This is a further check of the need of a holonomy group of $M$. We will adopt a unified notation for this projector in four and eight dimensions, with the convention that $C$ is just the unity in four dimensions and the octonionic 4-form in eight dimensions :

$$
w_{2}^{-} \equiv \frac{2}{n}\left(w_{2}-\star C w_{2}\right)
$$

\subsection{Expression of the $s$ and $\delta$ symmetries}

The resolution of the horizontality condition has thus given us the following expression for the action $s$ and $\delta$ on the fields we started from, plus the fields that we had to introduce to solve the degeneracies. These transformation laws are written in a way that is globally well defined:

$$
\begin{array}{ll}
s_{c} A=\Psi & \delta_{\bar{c}} A=g(\kappa) \eta+i_{\kappa} \chi \\
s_{c} \Psi=-d_{A} \Phi & \delta_{\bar{c}} \Psi=i_{\kappa}(T+F)+g(\kappa)[\Phi, \bar{\Phi}] \\
s_{c} \Phi=0 & \delta_{\bar{c}} \Phi=i_{\kappa} \Psi \\
s_{c} \bar{\Phi}=\eta & \delta_{\bar{c}} \bar{\Phi}=0 \\
s_{c} \eta=[\Phi, \bar{\Phi}] & \delta_{\bar{c}} \eta=\mathscr{L}_{\kappa} \bar{\Phi} \\
s_{c} \chi=T & \\
s_{c} T=[\Phi, \chi] & \delta_{\bar{c}} \chi=\frac{n}{2}\left(g(\kappa) d_{A} \bar{\Phi}\right)^{-} \\
\delta_{\bar{c}} T=-\frac{n}{2}\left(g(\kappa) d_{A} \eta+g(\kappa)[\bar{\Phi}, \Psi]\right)^{-}+\mathscr{L}_{\kappa} \chi
\end{array}
$$




$$
\begin{array}{ll}
s c=\Phi-c^{2} & \delta c=i_{\kappa}(A-\stackrel{\circ}{A})-|\kappa| b \\
s \bar{c}=b-[c, b] & \delta \bar{c}=|\kappa|\left(\bar{\Phi}-\bar{c}^{2}\right) \\
s b=[\Phi, \bar{c}]-[c, b] & \delta b=\mathscr{L}_{\kappa} \bar{c}-|\kappa| \eta
\end{array}
$$

Let us give for the sake of clarity the explicit $c$ and $\bar{c}$ dependence in the transformation laws Eqs. (66)

$$
\begin{aligned}
& s A=\Psi-d_{A} c \quad \delta A=g(\kappa) \eta+i_{\kappa} \chi-|\kappa| d_{A} \bar{c} \\
& s \Psi=-d_{A} \Phi-[c, \Psi] \quad \delta \Psi=i_{\kappa}(T+F)+g(\kappa)[\Phi, \bar{\Phi}]-|\kappa|[\bar{c}, \Psi] \\
& s \Phi=-[c, \Phi] \quad \delta \Phi=i_{\kappa} \Psi-|\kappa|[\bar{c}, \bar{\Phi}] \\
& s \bar{\Phi}=\eta-[c, \bar{\Psi}] \quad \delta \bar{\Phi}=-|\kappa|[\bar{c}, \bar{\Psi}] \\
& s \eta=[\Phi, \bar{\Phi}]-[c, \eta] \quad \delta \eta=\mathscr{L}_{\kappa} \bar{\Phi}-|\kappa|[\bar{c}, \eta] \\
& s \chi=T-[c, \chi] \quad \delta \chi=\frac{n}{2}\left(g(\kappa) d_{A} \bar{\Phi}\right)^{-}-|\kappa|[\bar{c}, \chi] \\
& s T=[\Phi, \chi]-[c, T] \quad \delta T=-\frac{n}{2}\left(g(\kappa) d_{A} \eta+g(\kappa)[\bar{\Phi}, \Psi]\right)^{-}+\mathscr{L}_{\kappa} \chi-|\kappa|[\bar{c}, T]
\end{aligned}
$$

The following remarks are important :

- As for an explicit dependence on the background connection, it only occurs in $\delta c$.

- The part of the BRST algebra that is relevant for untwisting only involve the fields in the first sector of the BRST equations, in Eqs. (66). It is important to note that the BRST invariance, introduces the Faddeev-Popov ghost in a way that is compatible with the $\delta$ invariance. This property is, in particular, very important for the questions relative to renormalization of supersymmetric gauge theories.

- In the flat space limit, we can define the anticommuting generators $s_{\mu}$, using $\delta_{\bar{c}}=$ $\kappa^{\mu} s_{\mu}$, and expanding in $\kappa^{\mu}$, both in 4 and 8 dimensions. The action of $s_{\mu}$ for the fields in (68) identifies itself with the vector symmetry [9] used in $d=4$ and obtained by twisting the supersymmetry algebra. However, here, the presence of auxiliary fields and Faddeev-Popov ghosts and antighost fully ensures consistency.

- The fields $\bar{c}$ and $b$ are indispensable in order to close the $s$ and $\delta$ operator on the Fadeev-Popov ghost $c$. In fact, the existence of the $\delta$ symmetry, and its link with reparametrization invariance, is likely to provide the geometrical interpretation of $\bar{c}$ and $b$, as well as of other antighosts of the TQFT. 
- The ghost antighost duality which will be defined in the next section will make the latter point more precise.

- Power counting arguments based on the dimensionality and ghost number of fields show that the above transformation laws are the most general ones that solve the relations

$s^{2}=\delta^{2}=0,\{s, \delta\}=\stackrel{\circ}{\mathscr{L}}_{\kappa}$, up to irrelevant field multiplicative renormalization factors and linear field redefinitions for the "auxiliary" fields $b$ and $T$. The geometrical horizontality equation that we defined in the fiber bundle actually solve this constraint.

In the next section, we will construct invariant actions for the symmetry. Eventually, we will discuss this untwisting toward the Poincaré superalgebra.

\section{The action}

\subsection{The topological/supersymmetric action as a $s$-exact term}

We now have a complete realization of the $s$ and $\delta$ symmetries, and wish to compute an invariant action.

Even if the algebra is defined only on a manifold of reducible tangent bundle, we demand a $\operatorname{Spin}(7)$ or an $S O(4)$ invariant action in respectively eight and four dimensions. This means that the action must be independent of $\kappa$, which is an non trivial requirement. (Our construction needs a base space of the topological Yang-Mills theory that contains a constant vector. However, we wish the theory be generalizable for a more general manifold, provided it has the required holonomy for defining self-duality equations.)

We will focus on the terms of power counting corresponding to the strictly renormalizable case in four dimensions, and extend this requirement in 8 dimensions, which can be done by a formal power counting argument (so we exclude higher order derivative terms).

The only way to construct an $\hat{\mathcal{S}}$-exact action, which is invariant under gauge transformations, reparametrization invariant and independent of $\kappa$, is such that

$$
\begin{array}{r}
S=s \Psi^{(-1,0)}+\delta \Psi^{(0,-1)} \\
\delta \Psi^{(-1,0)}=0 \quad s \Psi^{(0,-1)}=0
\end{array}
$$

There is only one solution of $s \Psi^{(0,-1)}=0$ which gives an action independent of $\kappa$. In turn, the $\delta$-invariance completely determines $\Psi^{(-1,0)}$ (with the hypothesis that there are nor higher order derivative terms). As a matter of fact, up to a global scale factor and a 
topological term, these two solutions give the same action. One has indeed :

$$
s \Psi^{(-1,0)}=\delta \Psi^{(0,-1)}+\frac{1}{2} \int_{M} C_{\wedge} \operatorname{Tr} F_{\wedge} F
$$

So, we restrict ourself to the $\delta$ invariant gauge function, and we define :

$$
S=s \Psi
$$

where $\Psi$ is a $\delta$-closed gauge invariant functional, such that :

$$
\Psi=\int_{M} \operatorname{Tr}\left(\chi \star\left(F+\frac{2}{n} T\right)+\bar{\Phi} d_{A} \star \Psi+\star \eta[\Phi, \bar{\Phi}]\right)
$$

One has the usual interpretation that the action $s \Psi$ is the gauge-fixing of a topological invariant. But the gauge fixing-function has been fixed from $\delta_{\bar{c}}$ invariance.

We will shortly show that there is a duality symmetry between $s$ and $\delta$, and that one can express this action as a $s \delta$-exact action.

\subsection{Gauge-fixing part}

We identify $\bar{c}$ and $b$ as the familiar Faddeev-Popov pair of an antighost and a Lagrange multiplier for gauge-fixing the Yang-Mills invariance. We could use a term like $s(\bar{c} \partial \cdot A)$, which violates the $\delta$-invariance, but we prefer a $s \delta$-exact term:

$$
s \delta \frac{1}{2|\kappa|} \int_{M} \operatorname{Tr}((A-\stackrel{\circ}{A}) \star(A-\stackrel{\circ}{A}))
$$

This gauge-fixing term breaks the $S O(n)$ invariance, since it depends on $\kappa$. From the point of view of the equivariant theory, however, the relevant action is the part of the action that is gauge invariant. It is determined by both $s$ and $\delta$ invariance. A further $S O(n)$ invariant gauge-fixing of the yang-Mills symmetry implies the breaking of the vector symmetry. (This is of course a gauge-fixing artifact, which does not appear for the gauge invariant observables, which are $\kappa$-independent). This is understandable in the untwisted formalism, where a supersymmetric gauge-fixing process of the Yang-Mills invariance only holds in a fully superspace version of the theory, and would yield an infinite number of fields in 8 dimensions. ${ }^{4}$

4 A refinement of our work can be reached by extending our result in the context of equivariant invariance, which implies the introduction of a background gauge symmetry, along the line of [16, 17]. 


\subsection{A $s \delta$-exact expression of the supersymmetric action}

As in the case of $\mathcal{N}_{T}=2$ topological theories, the action (69) is $s \delta$-exact on a reducible manifold. Indeed, we can verify the following very suggestive formula, which shows directly $s$ and $\delta$ invariances :

$$
S=s \delta \int_{M} \frac{1}{|\kappa|}{ }^{2} \mathscr{F}
$$

with

$$
\left.\mathscr{F}=\operatorname{Tr}\left(\frac{1}{2} g(\kappa)_{\wedge} C_{\wedge}\left((A-\stackrel{\circ}{A})_{\wedge}(F+\stackrel{\circ}{F})-\frac{1}{3}(A-\stackrel{\circ}{A})^{3}\right)+\left(g(\kappa) \eta+i_{\kappa} \chi\right)\right)_{\wedge} \Psi\right)
$$

where we recognize the last term to be the Chern-Simon term

$$
d \operatorname{Tr}\left((A-\stackrel{\circ}{A})_{\wedge}(F+\stackrel{\circ}{F})-\frac{1}{3}(A-\stackrel{\circ}{A})^{3}\right)=\operatorname{Tr}\left(F_{\wedge} F-\stackrel{\circ}{F} \stackrel{\circ}{F}^{3}\right)
$$

Note that in the case of a trivial fiber bundle we can take $\stackrel{\circ}{A}=0$ and recover the ordinary form of the Chern-Simon term. Unlike in the $\mathcal{N}_{T}=2$ case, the "potential" $\int_{M} \mathscr{F}$ cannot be interpreted as a Morse function for the theory. Indeed, the function $\int_{M} \operatorname{Tr}\left(\frac{1}{2} g(\kappa){ }_{\wedge} C_{\wedge}\left(A d A+\frac{2}{3} A^{3}\right)\right)$ has degenerated critical points, $\left(i_{\kappa} A\right.$ does not appear in its equations of motions), while the Chern-Simons potential gives a well defined Morse function in dimension $n-1$, when there is a balanced topological theories [13].

\subsection{Ghost antighost duality}

In spite of the fact that $\int_{M} \mathscr{F}$ is not a well defined Morse function, we can interpret formally

$$
F+g(\kappa) \eta+i_{\kappa} \chi+|\kappa|^{2} \bar{\Phi}
$$

as a curvature as in the case $\mathcal{N}_{T}=2$. This interpretation can be seen by introducing an involution $*$, which gives a duality between ghosts and anti-ghosts.

$$
\begin{gathered}
* A=A \quad * T=-T-\frac{n}{2|\kappa|^{2}}\left(g(\kappa) i_{\kappa} F\right)^{-} \\
* \Psi=g(\kappa) \eta+i_{\kappa} \chi \quad * \eta=\frac{1}{|\kappa|}{ }_{2} i_{\kappa} \Psi \quad * \chi=\frac{n}{2|\kappa|^{2}}(g(\kappa) \Psi)^{-} \\
* \Phi=|\kappa|^{2} \bar{\Phi} \quad * \bar{\Phi}=\frac{1}{|\kappa|}{ }^{2} \Phi \\
* c=|\kappa| \bar{c} \quad * b=-b+[c, \bar{c}]+\frac{1}{|\kappa|} i_{\kappa}(A-\stackrel{\circ}{A}) \quad * \bar{c}=\frac{1}{|\kappa|} c
\end{gathered}
$$


This involution relates both operators $s$ and $\delta$, as follows :

$$
* s *=\delta \quad * \delta *=s
$$

Note that $*$ does not act as a derivative but as a group element, that is

$$
*(A \cdot B)=* A \cdot * B
$$

Indeed, by definition of $*$, we can associate to the $\delta$-invariant gauge function $\Psi$ defined in Eq. (72) a mirror $s$-invariant gauge function, which defines a slightly different $\delta$-exact gauge invariant action $* S$, which gives the same observables. By construction, $* S$ is $s$ - and $\delta$-invariant. However $* S$ is $\kappa$-dependent, but this dependence disappears after elimination of the auxiliary field $T$, and then $* S$ becomes equal to $S$.

The horizontality relation gives indeed :

$$
\begin{gathered}
(d+\delta)(A+|\kappa| \bar{c})+(A+|\kappa| \bar{c})^{2}=F+g(\kappa) \eta+i_{\kappa} \chi+|\kappa|^{2} \bar{\Phi} \\
\left(d_{A}+\delta_{\bar{c}}\right)\left(F+g(\kappa) \eta+i_{\kappa} \chi+|\kappa|^{2} \bar{\Phi}\right)=0
\end{gathered}
$$

The gauge function $* \Psi$ replaces the ordinary gauge function $\Psi$, when one interchanges the rôles of $s$ and $\delta$ :

$$
* \Psi=\left.\frac{1}{|\kappa|}\right|^{2} \int_{M} \operatorname{Tr}\left(-g(\kappa)_{\wedge} C_{\wedge} \Psi_{\wedge} F-g(\kappa) \Psi \star T+\Phi d_{A} \star\left(g(\kappa) \eta+i_{\kappa} \chi\right)+\star i_{\kappa} \Psi[\bar{\Phi}, \Phi]\right)
$$

Eventually, we can define the topological observables as functions of the dual variables, using any gauge invariant polynomial in the fields $P(F, \Psi, \Phi)$, as follows :

$$
\begin{aligned}
*\langle P(F, \Psi, \Phi)\rangle & =* \int \mu P(F, \Psi, \Phi) e^{-s \Psi} \\
& =\int * \mu P\left(F, g(\kappa) \eta+i_{\kappa} \chi,|\kappa|^{2} \bar{\Phi}\right) e^{-\delta * \Psi} \\
& =\left\langle P\left(F, g(\kappa) \eta+i_{\kappa} \chi,|\kappa|^{2} \bar{\Phi}\right)\right\rangle
\end{aligned}
$$

The descent equations are obtained by changing $d+s$ into $d+\delta$. As a conclusion, after the duality operation $*$, the curvature of the "big bundle" defined in [19] becomes $F+g(\kappa) \eta+i_{\kappa} \chi+|\kappa|^{2} \bar{\Phi}$ instead of $F+\Psi+\Phi$.

\section{Conservation of the energy-momentum tensor an- tecedent $\Lambda_{\mu \nu}$}

We now verify that the operator $\delta$ is truly generated by the conservation law of the BRST antecedent of the energy momentum tensor. This computation is tricky, since the 
topological action is generally only invariant under $G \subset S O(n)$. Let us write the action $s \Psi$ as :

$$
\begin{aligned}
S=\int_{M} d^{n} x \sqrt{g} \operatorname{Tr}\left(\frac{1}{2} T^{\mu \nu}\left(-F_{\mu \nu}+\frac{2}{n} T_{\mu \nu}\right)+\chi^{\mu \nu}\left(D_{\mu} \Psi_{\nu}-\frac{1}{n}\left[\Phi, \chi_{\mu \nu}\right]\right)\right. \\
\left.+\eta D_{\mu} \Psi^{\mu}+\bar{\Phi}\left\{\Psi_{\mu}, \Psi^{\mu}\right\}-\bar{\Phi} D_{\mu} D^{\mu} \Phi+[\Phi, \bar{\Phi}]^{2}-\eta[\Phi, \eta]\right)
\end{aligned}
$$

To compute the energy momentum tensor, we use the standard formula :

$$
T_{\mu \nu}=\frac{1}{e} \delta_{a b} \frac{\delta S}{\delta e_{b}^{\mu}} e_{\nu}^{a}
$$

We have to understand the way the projector on self-dual 2-forms transform under variations of the vielbeins. In 8 dimensions, the variation of this projector $P^{-}$with respect to the vielbeins is ${ }^{5}$ :

$$
\begin{aligned}
& \delta P_{\mu \nu}^{-\sigma \rho}=-\frac{1}{n} \delta\left(e_{\mu}^{a} e_{\nu}^{b} e_{c}^{\sigma} e_{d}^{\rho}\right) C_{a b}{ }^{c d}
\end{aligned}
$$

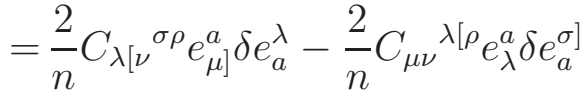

Using this formula, the energy momentum tensor is given by

$$
\begin{array}{r}
T_{\mu \nu}=\operatorname{Tr}\left(-T_{\nu}{ }^{\sigma} F_{\mu \sigma}^{+}+2 \chi_{\nu}{ }^{\sigma} D_{[\mu} \Psi_{\sigma]_{+}}-2 D_{\{\mu} \eta \cdot \Psi_{\nu\}}+2 \bar{\Phi}\left\{\Psi_{\mu}, \Psi_{\nu}\right\}+2 D_{\{\mu} \bar{\Phi} \cdot D_{\nu\}} \Phi\right. \\
+g_{\mu \nu}\left(\left(\frac{2}{n}-\frac{1}{2}\right) T^{\sigma \rho}\left(-F_{\sigma \rho}+\frac{2}{n} T_{\sigma \rho}\right)+\left(\frac{4}{n}-1\right) \chi^{\sigma \rho}\left(D_{\sigma} \Psi_{\rho}-\frac{1}{n}\left[\Phi, \chi_{\sigma \rho}\right]\right)\right. \\
\left.\left.+D_{\sigma} \eta \cdot \Psi^{\sigma}-\bar{\Phi}\left\{\Psi_{\sigma}, \Psi^{\sigma}\right\}-D_{\sigma} \bar{\Phi} \cdot D^{\sigma} \Phi-[\Phi, \bar{\Phi}]^{2}+\eta[\Phi, \eta]\right)\right)
\end{array}
$$

This tensor is not symmetric in eight dimensions and its antisymmetric part is antiselfdual (it is valued in $\mathfrak{s o}(8) \backslash \mathfrak{s p i n}(7)$ ). This is allowed to the fact that only isometries which preserve the octonionic 4-form $C$ define conserved charges. In four dimensions, it is symmetric.

Since the BRST operator does not depend on $e_{\mu}^{a}$, we have :

$$
T_{\mu \nu}=s \frac{1}{e} \delta_{a b} \frac{\delta \Psi}{\delta e_{b}^{\mu}} e_{\nu}^{a}
$$

\footnotetext{
${ }^{5}$ In 4 dimensions, one replaces $C_{a b}{ }^{c d}$ by the $\epsilon$ symbol
} 
In this way, we find a $s$-antecedent of the energy momentum tensor :

$$
\begin{aligned}
\Lambda_{\mu \nu}^{(0)}=g_{\mu \nu} \operatorname{Tr}\left(\frac{1}{2}\left(\frac{4}{n}-1\right) \chi^{\sigma \rho}\right. & \left.\left(-F_{\sigma \rho}^{-}+\frac{2}{n} T_{\sigma \rho}\right)+\Psi^{\sigma} D_{\sigma} \bar{\Phi}-\eta[\Phi, \bar{\Phi}]\right) \\
& -\operatorname{Tr}\left(\chi_{\nu}{ }^{\sigma} F_{\mu \sigma}^{+}+2 \Psi_{\{\mu} D_{\nu\}} \bar{\Phi}\right)
\end{aligned}
$$

It is not yet conserved. However, by adding a $s$-exact term to $\Lambda_{\mu \nu}^{(0)}$, we can enforce the conservation law, as follows:

$$
\begin{gathered}
\nabla^{\nu} \Lambda_{\mu \nu} \equiv \nabla^{\nu}\left(\Lambda_{\mu \nu}^{(0)}-s \operatorname{Tr}\left(\frac{1}{2} C_{\mu \nu}^{\star{ }^{\sigma \rho}} F_{\sigma \rho} \bar{\Phi}+\chi_{\mu \nu} \eta-\frac{1}{2}\left(1-\frac{4}{n}\right) \chi_{[\mu}{ }^{\sigma} \chi_{\nu] \sigma}\right)\right) \\
=-\operatorname{Tr}\left(\left(-g_{\mu \nu} \eta+\chi_{\mu \nu}\right) \frac{\delta^{L} S}{\delta A_{\nu}}+\left(F_{\mu \nu}-T_{\mu \nu}+g_{\mu \nu}[\Phi, \bar{\Phi}]\right) \frac{\delta^{L} S}{\delta \Psi_{\nu}}-\Psi_{\mu} \frac{\delta^{L} S}{\delta \Phi}\right. \\
+D_{\mu} \bar{\Phi} \frac{\delta^{L} S}{\delta \eta}-n D^{\nu} \bar{\Phi} \frac{\delta^{L} S}{\delta \chi^{\mu \nu}}+\frac{n}{2}\left(2 D^{\nu} \eta+D_{\sigma} \chi^{\sigma \nu}-2\left[\bar{\Phi}, \Psi^{\nu}\right]\right) \frac{\delta^{L} S}{\delta T^{\mu \nu}} \\
\left.-\frac{n}{2} \chi^{\mu \sigma} D^{\nu} \frac{\delta^{L} S}{\delta T^{\nu \sigma}}+\left(\frac{n}{2}-2\right) D^{\nu}\left(\chi_{\mu}{ }^{\sigma} \frac{\delta^{L} S}{\delta T^{\nu \sigma}}\right)\right)
\end{gathered}
$$

From the above equation we can recover the explicit form of the functional generators $V_{A \mu}$ that we introduced in section 2 . We can verify that the resulting symmetry truly correspond to the $\delta$-operator that we build in section 3.2 directly from the horizontality condition.

\section{Untwisting toward Yang-Mills supersymmetry}

The theories that we have determined in four and eight dimensions from $\delta$ and $s$ invariances correspond by twist to superYang-Mills $\mathcal{N}=2$ theories. The equivariant form of the scalar and the $\delta$ BRST operators can in fact be identified on twisted combinations of spinorial supersymmetry generators. As a matter of fact, if we define the theory on a manifold that is sufficiently constrained to admit two supersymmetries of opposed chirality, the equivariant form of these $s$ and $\delta$ can be related to supersymmetry transformations as follows:

$$
\theta s_{c}=\delta_{\text {susy }}(\theta \zeta) \quad \theta \delta_{\bar{c}}=\delta_{\text {susy }}(i \theta \text { 肪 } \zeta)
$$

for $\theta$ an anticommuting parameter and $\zeta$ a chiral covariantly constant spinor. As it is well known, in eight dimensions we can construct the $\mathcal{N}=2$ superYang-Mills algebra from 
the dimensional reduction on a torus of the ten dimensional superYang-Mills algebra, with a further Wick rotation of the $\mathcal{N}=1$ theory that is generally defined on Minkowski space. The transition from $\mathcal{N}=1$ to $\mathcal{N}=2$ is allowed by the fact that the MajoranaWeyl condition is consistent in eight dimensions for an Euclidean space and not for a Minkowski one. The Euclidean action obtained in this way is

$$
\begin{aligned}
\int_{M} d^{8} x \sqrt{g} \operatorname{Tr}\left(-\frac{1}{4} F_{\mu \nu} F^{\mu \nu}+\right. & \frac{1}{2} D_{\mu} \phi_{1} D^{\mu} \phi_{1}-\frac{1}{2} D_{\mu} \phi_{2} D^{\mu} \phi_{2}-\frac{i}{2}(\bar{\lambda} \not D \lambda) \\
& \left.+\frac{1}{2}\left[\phi_{1}, \phi_{2}\right]^{2}+\frac{1}{2}\left(\bar{\lambda} \gamma_{9}\left[\phi_{1}, \lambda\right]\right)-\frac{1}{2}\left(\bar{\lambda}\left[\phi_{2}, \lambda\right]\right)\right)
\end{aligned}
$$

It is invariant under the following supersymmetries for any covariantly constant spinor $\epsilon$

$$
\begin{aligned}
\delta_{\text {susy }} A_{\mu} & =-i\left(\bar{\epsilon} \gamma_{\mu} \lambda\right) \\
\delta_{\text {susy }} \phi_{1} & =-\left(\bar{\epsilon} \gamma_{9} \lambda\right) \\
\delta_{\text {susy }} \phi_{2} & =-(\bar{\epsilon} \lambda) \\
\delta_{\text {susy }} \lambda & =\not F \epsilon-i \gamma_{9} \not D \phi_{1} \epsilon-i \not D \phi_{2} \epsilon+\gamma_{9}\left[\phi_{1}, \phi_{2}\right] \epsilon
\end{aligned}
$$

If $M$ is defined to be a $\operatorname{Spin}(7)$-manifold, it contains a chiral covariantly constant spinor $\zeta$. We choose it Majorana-Weyl with norm equal to one. One can further construct the octonionic 4-form, as follows:

$$
(\bar{\zeta} \zeta)=1 \quad 4 !\left(\bar{\zeta} \gamma_{\mu \nu \sigma \rho} \zeta\right)=C_{\mu \nu \sigma \rho}
$$

We can decompose the Majorana spinor fields of the theory in term of differential forms, by projection over $\zeta$, which is the definition of the twist in 8 dimensions:

$$
\begin{aligned}
& \lambda_{+}=(\eta+\chi) \zeta \\
& \lambda_{-}=i \Psi \zeta
\end{aligned}
$$

Here $\eta, \chi$ and $\Psi$ represent the same fields as in the previous sections, and the convention for the crossed out forms of rank $k$ is that they are contracted with $k$ gamma matrices with a normalization factor $\frac{1}{k !}$. Then, we have the redefinition for the scalar fields:

$$
\Phi \equiv-\left(\phi_{1}-\phi_{2}\right) \quad \bar{\Phi} \equiv-\frac{1}{2}\left(\phi_{1}+\phi_{2}\right)
$$

The twisted action that one obtains in this way is

$$
\begin{aligned}
\int_{M} d^{8} x \operatorname{Tr}\left(-\frac{1}{4} F_{\mu \nu} F^{\mu \nu}+\eta D_{\mu} \Psi^{\mu}+D_{\mu} \bar{\Phi} D^{\mu} \Phi+4 \chi^{\mu \nu} D_{\mu} \Psi_{\nu}\right. & \\
& \left.+2 \bar{\Phi} \Psi_{\mu} \Psi^{\mu}+2 \Phi \chi_{\mu \nu} \chi^{\mu \nu}+\Phi \eta^{2}+\frac{1}{2}[\Phi, \bar{\Phi}]^{2}\right)
\end{aligned}
$$


It is the same action as that obtained in section 4, from the demand of $s$ and $\delta$ invariances, after the integration of the auxiliary field $T$, modulo some rescalings, and up to the sum of a topological term:

$$
-\frac{1}{2} \int_{M} C_{\wedge} \operatorname{Tr}\left(F_{\wedge} F\right)
$$

By using the decomposition by twist of the spinorial supersymmetry parameter $\epsilon=$ $\left(\theta+\Theta^{\mu \nu} \gamma_{\mu \nu}+i \vartheta^{\mu} \gamma_{\mu}\right) \zeta$, one gets twisted generators $Q, Q_{\mu}, Q_{\mu \nu}$. ( $Q$ and $\kappa^{\mu} Q_{\mu}$ are truly identified with the BRST operators $s_{c}$ and $\delta_{\bar{c}}$, in their equivariant form, both in 8 and 4 dimensions.)

Both charges $(\bar{\zeta} \mathcal{Q})$ and $i(\bar{\zeta} \nprec \mathcal{Q})$ completely constrain the supersymmetric theory. In this sense, the number of relevant supersymmetries can be reduced to five real supercharges in four dimensions (as already observed in [9]) and to nine real supercharges in in eight dimensions.

It is very instructive that this reduced number of relevant generators can be directly constructed from one extended horizontality condition, defined in the Yang-Mills principal bundle.

As a further remark, the tensor generator of supersymmetry cannot be closed off-shell in eight dimension [20], contrarily to the case of 4 dimensions ${ }^{6}$. It is unknown if a system of auxiliary fields can be introduced to close the algebra of maximal supersymmetry. The existence of the tensor symmetry is not foreseen from the point of view of TQFT's, and its existence seems unnecessary, since the $Q$ and $Q_{\mu}$ symmetries are enough to determine the supersymmetry action.

\section{Equivariant Topological Field Theories}

The observables of the topological theories that we discussed so far are defined as classes of the ordinary de Rham cohomology of the extended exterior derivative $\tilde{d}=d+s$ acting on $M \times \tilde{\mathcal{B}}^{*}$, where $M$ is the manifold on which the topological theory is formulated and $\tilde{\mathcal{B}}^{*}$ is the space of gauge orbits of irreducible framed connections $[19,26]$. In these theories, the scalar ghost-for-ghost field $\Phi$ goes to zero at infinity, or in other words,

\footnotetext{
${ }^{6}$ From a technical point of view, the difference between four and eight dimensions amounts the fact that one can construct an antiselfdual 2-form as a bilinear combination of two other in four dimensions, by the use of

$$
P^{-}{ }_{\mu \nu}^{\theta \tau} P^{-}{ }_{\theta}^{\eta \sigma \rho} P^{-}{ }_{\tau \eta}{ }^{\kappa \lambda}
$$

but not in eight, because this term is zero in this case.
} 
has vanishing vacuum expectation value. Since, as we can see from (96), the $\Phi$ field is related to the scalar fields of the supersymmetric Yang-Mills theory, it is interesting to construct a topological theory whose scalar fields acquire a non-vanishing vacuum expectation value [27]. This topological field theory can be obtained by considering the equivariant extension of the construction of [26] with respect to the Lie algebra $\mathfrak{h}$ of an Abelian subgroup $H \subset G$ acting on $\tilde{\mathcal{B}}^{*}$. For example, for $G=S U(N)$ one can consider the maximal Abelian subgroup $H=U(1)^{N-1}$, which is the suitable choice for the Seiberg-Witten model. Moreover, we also consider an equivariant extension with respect to a compact Abelian group of isometries $K$ of $M$. This corresponds to a kind of spontaneous breaking of the symmetries of $M$ down to $K$. In fact, as we will see in the following, the resulting equivariant topological theory corresponds to the twisted version of the Super Yang-Mills theory on a non-trivial gravitational background.

The equivariant formulation allows for the use of a powerful localization formula [28] that reduce the integral over the equivariant forms on $M \times \tilde{\mathcal{B}}^{*}$ to a sum over the isolated fixed points of the $K \times H$ symmetry $^{7}$. The results on the ordinary cohomology may in general be recovered by sending to zero the parameters associated to this symmetry with a suitable prescription. In this sense, the equivariant extension can be thought as a very useful regularization procedure for the topological invariants. The localization formula has been extended for supermanifolds in [29] and exploited in the four dimensional case to compute the integral on the instanton moduli space, recovering the non-perturbative contribution to the low-energy Seiberg-Witten effective action [29, 30, 31].

In the following we will discuss the equivariant extension of our horizontality conditions and obtain from them the scalar and vector topological symmetries along the same lines of the previous sections. Then we will untwist our topological theory and show its relationship with the supersymmetric theory on the $\Omega$-background introduced in [22].

\subsection{Equivariant horizontality condition}

Let us define the Weil complex corresponding to the equivariant cohomology

$$
H_{K \times H}^{\bullet}\left(M \times \tilde{\mathcal{B}}^{*}\right)
$$

equivariant with respect to the action of $K \times H$ on $\tilde{\mathcal{B}}^{*}$. The action of $K$ on $\tilde{\mathcal{B}}^{*}$ can be defined as follows. The action of $K$ on $M$ can be lifted to an action on the principal

\footnotetext{
${ }^{7}$ Notice that the fixed points are isolated only in this case: considering the equivariance with respect to only one of the two groups, $K$ or $H$, is not enough to localize to isolated points.
} 
bundle $P$ by the use of a background connexion $\stackrel{\circ}{A}$. This action induces a pullback action on the equivariant forms on $P$, which defines the action of $K$ on $\tilde{\mathcal{B}}^{*}$. The relevant equivariant differential $s_{\mathfrak{k}}$ on the Weil complex is defined as usual on an equivariant form $w(\xi)$ by

$$
\left(s_{\mathfrak{k}} w\right)(\xi) \equiv\left(s+I_{\xi^{*}}\right) w(\xi)
$$

where $\xi^{*}$ is the vector field of $T P$ generating the action on $\tilde{\mathcal{B}}^{*}$ associated to $\xi=(\Omega, a) \in$ $\mathfrak{k} \oplus \mathfrak{h} . \xi^{*}$ decomposes into the horizontal lift $v^{h}$ of the vector field $v$ of $T M$ generated by the element $\Omega$ of $\mathfrak{k}$ and the fundamental vector $a^{v}$ associated to the element $a$ of $\mathfrak{h}$,

$$
\xi^{*} \equiv\left(v^{h}, a^{v}\right) \in T H \oplus T V
$$

The closure of the equivariant BRST operator (100) reads on a generic form

$$
s_{\mathfrak{k}}^{2}=\mathcal{L}_{\xi^{*}}
$$

so that $s_{\mathfrak{k}}$ is a nilpotent operator on equivariant forms. This is locally expressed on $M$

$$
s_{\mathfrak{k}}^{2}=\mathcal{L}_{v}+\delta_{\text {gauge }}\left(i_{v} \stackrel{\circ}{A}-a\right)
$$

The explicit representation of the operator (100) on anti-self-dual gauge connections has been discussed in detail in $[31,29,32]$. Since $i_{\xi^{*}}$ commutes with $s_{\mathfrak{k}}$, we have the following nilpotent operator on the whole complex (not restricted to its invariant subcomplex)

$$
\left(d+s_{\mathfrak{k}}-i_{v^{h}+a^{v}}\right)^{2}=0
$$

So we can define $s_{\mathfrak{k}}$ as usual by the use of an horizontality condition

$$
\left(d+s_{\mathfrak{k}}-i_{v^{h}+a^{v}}\right)(A+c)+(A+c)^{2}=F+\Psi+\Phi
$$

and its associated Bianchi identity. Moving the term $i_{\xi^{*}} A$ to the right hand side we obtain the definition of the equivariant curvature [28]

$$
\left(d+s_{\mathfrak{k}}\right)(A+c)+(A+c)^{2}=F+\Psi+\Phi_{\mathfrak{k}}
$$

Notice that on the right hand side of (106) it appears the equivariant extension of the scalar field $\Phi_{\mathfrak{k}}=\Phi_{\mathfrak{h}}+i_{v}(A-\stackrel{\circ}{A})=\Phi+a+i_{v}(A-\stackrel{\circ}{A})$, where $\mu(\xi)=a+i_{v}(A-\stackrel{\circ}{A})$ is the moment of the vector field $\xi^{*}[28]$. This deformation of the scalar field is precisely that found in the explicit computations on the instanton moduli space in four dimensions [33]. The field $\Phi_{\mathfrak{h}}$ has a non-trivial vacuum expectation value in the Cartan subalgebra of the 
group $G$, due to the presence of the term $a[31,29,33]$. Notice that the vector $\xi^{*}$ has ghost number 2 .

The dual version of equation (105) is naturally defined with the use of another Killing vector $\bar{v}$ on $M$ and another element $\bar{a}$ of $\mathfrak{h}$ which define a vector $\bar{\xi}^{*}=\bar{v}^{h}+\bar{a}^{v}$ on $P$, all with ghost number -2 , and reads

$$
\left(d+\delta_{\mathfrak{k}}-|\kappa|^{2} i_{\bar{v}^{h}+\bar{a}^{v}}\right)(A+|\kappa| \bar{c})+(A+|\kappa| \bar{c})^{2}=F+g(\kappa) \eta+i_{\kappa} \chi+|\kappa|^{2} \bar{\Phi}
$$

Exactly in the same way as in the previous sections, we can combine the horizontality conditions (105) and (107) into a single one which will define the equivariant BRST operator as well as the corresponding vector symmetry. The extended horizontality condition is

$$
\begin{aligned}
\left(d+s_{\mathfrak{k}}+\delta_{\mathfrak{k}}-i_{\kappa^{h}+\xi^{*}+|\kappa|^{2} \bar{\xi}^{*}}\right)(A+c+|\kappa| \bar{c})+(A+c+|\kappa| \bar{c})^{2} & \\
& =F+\Psi+g(\kappa) \eta+i_{\kappa^{h}} \chi+\Phi+|\kappa|^{2} \bar{\Phi}
\end{aligned}
$$

and its associated Bianchi relation

$$
\begin{aligned}
&\left(d+s_{\mathfrak{k}}+\delta_{\mathfrak{k}}-i_{\kappa^{h}+\xi^{*}+|\kappa|^{2} \bar{\xi}^{*}}\right)\left(F+\Psi+g(\kappa) \eta+i_{\kappa^{h}} \chi+\Phi+|\kappa|^{2} \bar{\Phi}\right) \\
&+\left[A+c+|\kappa| \bar{c}, F+\Psi+g(\kappa) \eta+i_{\kappa^{h}} \chi+\Phi+|\kappa|^{2} \bar{\Phi}\right]=0
\end{aligned}
$$

By following the same procedure described in the previous sections for the ordinary topological field theory, and redefining now

$$
s_{c} \equiv s_{\mathfrak{k}}+\delta_{\text {gauge }}(c) \quad \delta_{\bar{c}} \equiv \delta_{\mathfrak{k}}+\delta_{\text {gauge }}(|\kappa| \bar{c})
$$

we can extract from (108) and (109) the complete transformations of the fields

$$
\begin{array}{lll}
s_{c} A=\Psi & \delta_{\bar{c}} A=g(\kappa) \eta+i_{\kappa} \chi \\
s_{c} \Psi=-d_{A} \Phi+i_{v} F & \delta_{\bar{c}} \Psi=i_{\kappa}(T+F)+g(\kappa) s_{c} \eta \\
s_{c} \Phi=i_{v} \Psi & \delta_{\bar{c}} \Phi=i_{\kappa} \Psi+(\kappa \cdot v) \eta+i_{v} i_{\kappa} \chi \\
s_{c} \bar{\Phi}=\eta+i_{\bar{v}} \Psi & \\
s_{c} \eta=[\Phi, \bar{\Phi}]+\mathscr{L}_{v} \bar{\Phi}-\mathscr{L}_{\bar{v}} \Phi+i_{\bar{v}} i_{v} F & \delta_{\bar{c}} \bar{\Phi}=(\bar{v} \cdot \kappa) \eta+i_{\bar{v}} i_{\kappa} \chi \\
& \delta_{\bar{c}} \eta=\mathscr{L}_{\kappa} \bar{\Phi}-i_{\kappa} i_{\bar{v}} F \\
s_{c} \chi=T & & \delta_{\bar{c} \chi}=\frac{n}{2}\left(g(\kappa)\left(d_{A} \bar{\Phi}-i_{\bar{v}} F\right)\right)^{-} \\
s_{c} T=[\Phi, \chi]+\mathscr{L}_{v} \chi & \delta_{\bar{c}} T=-\frac{n}{2}\left(g(\kappa)\left(d_{A} \eta+[\bar{\Phi}, \Psi]+\mathscr{L}_{\bar{v}} \Psi\right)\right)^{-}+\mathscr{L}_{\kappa} \chi
\end{array}
$$


We remark that in the four dimensional case the $s_{c}$ transformations on the fields in the first column of (111) induce exactly the BRST transformations on the instanton moduli space that have been used for the localization in [30, 31, 29]. The transformations in the Faddeev-Popov sector read

$$
\begin{array}{ll}
s_{\mathfrak{k}} c=\Phi+a-c^{2}+i_{v}(A-\stackrel{\circ}{A}) & \delta_{\mathfrak{k}} c=i_{\kappa}(A-\stackrel{\circ}{A})-|\kappa| b \\
s_{\mathfrak{k}} \bar{c}=b-[c, \bar{c}] & \delta_{\mathfrak{k}} \bar{c}=|\kappa|\left(\bar{\Phi}+\bar{a}-\bar{c}^{2}+i_{\bar{v}}(A-\stackrel{\circ}{A})\right) \\
s_{\mathfrak{k}} b=[\Phi, \bar{c}]-\mathscr{L}_{v} \bar{c}-[c, b] & \delta_{\mathfrak{k}} b=|\kappa|\left(\mathscr{L}_{\kappa} \bar{c}-\eta\right)
\end{array}
$$

The algebra (111), (112) closes off-shell, provided that $[v, \bar{v}]=\mathcal{L}_{v} \bar{v}=0, d(\kappa \cdot \bar{v})=0$. Moreover, $d g(v)$ and $d g(\bar{v})$ must be selfduals in the eight dimensional case. Then, one has, but on the Faddeev-Popov sector:

$$
\begin{gathered}
s_{c}^{2}=\delta_{\text {gauge }}\left(\Phi+i_{v} A\right)+\mathcal{L}_{v} \quad \delta_{\bar{c}}^{2}=\delta_{\text {gauge }}\left(\bar{\Phi}+i_{\bar{v}} A\right)+\mathcal{L}_{\bar{v}} \\
\left\{s_{c}, \delta_{\bar{c}}\right\}=\mathcal{L}_{\kappa}+\delta_{\text {gauge }}\left(i_{\kappa} A\right)
\end{gathered}
$$

The $s_{\mathfrak{k}}$ and $\delta_{\mathfrak{k}}$ symmetries completely constrain the classical action also in the equivariant case. The details of this computation are given in appendix B. The action of the equivariant topological theory is $s_{\mathfrak{k}} \delta_{\mathfrak{k}}$-exact

$$
S=s_{\mathfrak{k}} \delta_{\mathfrak{k}} \int_{M} \frac{1}{|\kappa|}{ }^{2} \mathscr{F}
$$

with

$$
\mathscr{F}=\operatorname{Tr}\left(\frac{1}{2} g(\kappa)_{\wedge} C_{\wedge}\left((A-\stackrel{\circ}{A})_{\wedge}(F+\stackrel{\circ}{F})-\frac{1}{3}(A-\stackrel{\circ}{A})^{3}\right)+\left(g(\kappa) \eta+i_{\kappa} \chi\right)_{\wedge} \star \Psi\right)
$$

and still displays an intriguing relationship with the Chern-Simons action functional. By acting with $\delta_{\mathfrak{k}}$ in (115) one gets

$$
\begin{aligned}
I=s_{\mathfrak{k}} \int_{M} \operatorname{Tr}\left(\chi \star\left(F+\frac{2}{n} T\right)+\Psi \star(\right. & \left.d_{A} \bar{\Phi}-i_{\bar{v}} F\right) \\
& \left.+\star \eta\left([\Phi, \bar{\Phi}]+\mathscr{L}_{v} \bar{\Phi}-\mathscr{L}_{\bar{v}} \Phi+i_{\bar{v}} i_{v} F\right)\right)
\end{aligned}
$$


and finally by acting with $s_{\mathfrak{k}}$

$$
\begin{aligned}
& I=\int_{M} \operatorname{Tr}\left(F^{-} \star F^{-}-\left(d_{A} \Phi-i_{v} F\right) \star\left(d_{A} \bar{\Phi}-i_{\bar{v}} F\right)+\chi \star d_{A} \Psi-\Psi \star d_{A} \eta\right. \\
&+T \star\left(F+\frac{2}{n} T\right)-\frac{2}{n} \chi \star\left([\Phi, \chi]+\mathscr{L}_{v} \chi\right)-\eta \star\left([\Phi, \eta]+\mathscr{L}_{v} \eta\right) \\
&\left.-\Psi \star\left([\bar{\Phi}, \Psi]+\mathscr{L}_{\bar{v}} \Psi\right)+\left([\Phi, \bar{\Phi}]+\mathscr{L}_{v} \bar{\Phi}-\mathscr{L}_{\bar{v}} \Phi+i_{\bar{v}} i_{v} F\right)^{2}\right)
\end{aligned}
$$

By comparing the equivariant topological action (117) to the topological action (97) one get a simple rule to pass from one to the other. In the case discussed in Sect. 4 the $s_{c}$ and $\delta_{\bar{c}}$ operators are nilpotent modulo gauge transformations, 1.e. $s_{c}^{2}=\delta_{\text {gauge }}(\Phi)$ and $\delta_{\bar{c}}^{2}=\delta_{\text {gauge }}(\bar{\Phi})$. In the equivariant case, the nilpotency is also verified modulo reparametrizations along the Killing vectors $v$ and $\bar{v}$, as one can see from the first line of Eq. (113). To pass from the ordinary topological theory to the equivariant one, we have to make the substitution

$$
\delta_{\text {gauge }}(\Phi) \rightarrow \delta_{\text {gauge }}\left(\Phi+i_{v} A\right)+\mathcal{L}_{v} \quad \delta_{\text {gauge }}(\bar{\Phi}) \rightarrow \delta_{\text {gauge }}\left(\bar{\Phi}+i_{\bar{v}} A\right)+\mathcal{L}_{\bar{v}}
$$

This amounts to the redefinitions

$$
\begin{gathered}
d_{A} \Phi \rightarrow d_{A} \Phi-i_{v} F \quad d_{A} \Phi \rightarrow d_{A} \bar{\Phi}-i_{\bar{v}} F \\
{[\Phi, \bar{\Phi}] \rightarrow[\Phi, \bar{\Phi}]+\mathscr{L}_{v} \bar{\Phi}-\mathscr{L}_{\bar{v}} \Phi+i_{\bar{v}} i_{v} F}
\end{gathered}
$$

for the bosonic fields, and

$$
\begin{gathered}
{[\Phi, \chi] \rightarrow[\Phi, \chi]+\mathscr{L}_{v} \chi \quad[\Phi, \eta] \rightarrow[\Phi, \eta]+\mathscr{L}_{v} \eta} \\
{[\Phi, \Psi] \rightarrow[\bar{\Phi}, \Psi]+\mathscr{L}_{\bar{v}} \Psi}
\end{gathered}
$$

for the fermion fields. One can check that, by doing the substitutions (119) and (120) in the topological action (97), one obtains the equivariant topological action (117).

Finally, we see that in the equivariant topological theory, the scalar field $\Phi_{\mathfrak{h}}$ has a nontrivial expectation value. In the following subsection we will show that the equivariant action (117) can be related by twist to the Super Yang-Mills theory on the $\Omega$-background introduced in [22].

\subsection{Dimensional reduction and $\Omega$ background}

The so-called $\Omega$-background can be introduced by considering a non-trivial dimensional reduction of the Super Yang-Mills theory on a torus. In this dimensional reduction, the 
original theory is defined on a Riemannian fiber bundle $E$

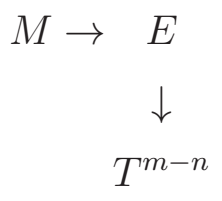

such that the manifold $M$ on which the dimensionally reduced theory lives is fibered on the torus. Eventually, we can define the metric as follows :

$$
G \equiv \delta_{\alpha \beta} d y^{\alpha} \otimes d y^{\beta}+g_{\mu \nu}\left(d x^{\mu}+v_{\alpha}^{\mu} d y^{\alpha}\right) \otimes\left(d x^{\nu}+v_{\beta}^{\nu} d y^{\beta}\right)
$$

Here, the $x^{\mu}$ are local coordinates on $M, y^{\alpha}$ are coordinates on $T^{m-n}$ and $v_{\alpha}$ are vector fields on $M$. It is natural to require that the metric $G$ does not depend on the torus coordinates; in fact, any non-trivial dependence would forbid a consistent cancellation of non-zero modes in the limit of zero radius.

In order to have a supersymmetric theory, one requires the existence of, at least, one supersymmetry generator, and thus the existence of a covariantly constant spinor field on the manifold. This implies that both manifolds $M$ and $E$ are Ricci flat [21] :

$$
{ }^{E} R_{m n}=0 \quad{ }^{M} R_{\mu \nu}=0
$$

These equations turn into constraints on the vector fields $v_{\alpha}$

$$
\begin{aligned}
\mathcal{L}_{v_{\alpha}} g_{\mu \nu} & =0 \\
{\left[v_{\alpha}, v_{\beta}\right] } & =0 \\
d \star d g\left(v_{\alpha}\right) & =0
\end{aligned}
$$

The first equation in (124) implies that $v_{\alpha}$ 's are Killing vectors for the manifold $M$, while the second imposes that they commute, i.e. $\mathcal{L}_{v_{\alpha}} v_{\beta}=0$. The vectors $v_{\alpha}$ can be mapped upon the Killing vectors that we used in the construction of the equivariant topological field theory in the previous subsection. The last equation in (124) imposes further restrictions on the $v_{\alpha}$ 's in order to preserve the supersymmetry. These conditions are not present in the topological theory. In fact, as we will see in detail in the following, they can be relaxed at the price of breaking the $S O(n)$ rotation invariance of the supersymmetric theory to the special holonomy subgroup required to define the topologically twisted theory.

Let us now work out the case of the eight-dimensional super Yang-Mills theory. 


\subsubsection{Supersymmetric formulation in eight dimensions}

The ten-dimensional vielbeins corresponding to the metric (122) are

$$
e_{m}^{A} \hat{=}\left(\begin{array}{cc}
e_{\mu}^{a} & i_{v_{\beta}} e^{a} \\
0 & \delta_{\beta}^{\alpha}
\end{array}\right) \quad e_{A}^{m} \hat{=}\left(\begin{array}{cc}
e_{a}^{\mu} & 0 \\
-v_{\alpha}^{\mu} & \delta_{\alpha}^{\beta}
\end{array}\right)
$$

The dimensional reduction of the ten-dimensional Yang-Mills curvature reads

$$
\frac{1}{2} F_{m n} d x^{m} \wedge_{\wedge} d x^{n}=\frac{1}{2} F_{\mu \nu} d x_{\wedge}^{\mu} d x^{\nu}+D_{\mu} \phi_{\alpha} d x^{\mu} \wedge_{\wedge} d y^{\alpha}+\frac{1}{2}\left[\phi_{\alpha}, \phi_{\beta}\right] d y_{\wedge}^{\alpha} d y^{\beta}
$$

We can write this curvature in locally flat coordinates by using the vielbeins (125)

$$
\begin{aligned}
\frac{1}{2} F_{A B} e^{A} \wedge e^{B}=\frac{1}{2} F_{a b} e^{a} \wedge_{\wedge} e^{b} & +\left(e_{a}^{\mu} D_{\mu} \phi_{\beta}-v_{\beta}^{\nu} e_{a}^{\mu} F_{\mu \nu}\right) e^{a} \wedge_{\wedge} d y^{\alpha} \\
& +\frac{1}{2}\left(\left[\phi_{\alpha}, \phi_{\beta}\right]-v_{\alpha}^{\mu} D_{\mu} \phi_{\beta}+v_{\beta}^{\mu} D_{\mu} \phi_{\alpha}+v_{\alpha}^{\mu} v_{\beta}^{\nu} F_{\mu \nu}\right) d y^{\alpha}{ }_{\wedge} d y^{\beta}
\end{aligned}
$$

By plugging (126) into the action

$$
\int_{M} d^{8} x \sqrt{g} \operatorname{Tr}\left(-\frac{1}{4} F_{A B} F^{A B}+\frac{i}{2}\left(\bar{\Lambda} \Gamma^{A} D_{A} \Lambda\right)\right)
$$

one can read the bosonic part of the eight-dimensional action. Concerning the fermionic part, we have to decompose the contraction of the covariant derivative $e_{A}^{m} D_{m}$. To simplify the computation we observe that

$$
e_{A}^{m} D_{m}=\mathscr{L}_{e_{A}^{m}}
$$

since $e_{A}^{m}$ is covariantly constant. The Lie derivative is independent of the Riemannian connection and thanks to this property, one has :

$$
e_{A}^{m} D_{m} \hat{=}\left(e_{a}^{\mu} D_{\mu}, \phi_{\alpha}-\mathscr{L}_{v_{\alpha}}\right)
$$

The ten-dimensional gamma matrices are related to those in eight dimensions as follows:

$$
\Gamma^{m} \equiv \sigma_{2} \otimes \gamma^{\mu}, \sigma_{2} \otimes \gamma_{9}, \sigma_{1} \otimes 1
$$

Using the above equations one obtains the action and its supersymmetries on a eight dimensional pseudo-Riemannian manifold. By extending to eight dimensions the Wick 
rotation on the fermions, which is defined in [23], one can Wick-rotate this theory to a Riemannian manifold, as follows :

$$
\begin{array}{cc}
x^{0} \rightarrow e^{-i \theta} x^{0} & \gamma^{8} \equiv i \gamma^{0} \\
A_{\mu} \rightarrow\left(e^{i \theta} A_{8}, A_{i}\right) & \\
\lambda \rightarrow e^{\frac{1}{2} \theta \gamma^{8} \gamma_{9}} \lambda & \lambda^{\dagger} \rightarrow \lambda^{\dagger} e^{\frac{1}{2} \theta \gamma^{8} \gamma_{9}} \\
\phi_{1} \rightarrow e^{i \theta} \phi_{1} & \phi_{2} \rightarrow \phi_{2} \\
v_{1} \rightarrow e^{i \theta} v_{1} & v_{2} \rightarrow v_{2}
\end{array}
$$

Eventually, one sets $\theta=\frac{\pi}{2}$, and one gets the Euclidean action

$$
\begin{aligned}
& \int_{M} d^{8} x \sqrt{g} \operatorname{Tr}\left(-\frac{1}{4} F_{\mu \nu} F^{\mu \nu}-\right. \frac{1}{2}\left(D_{\mu} \phi_{\alpha}+v_{\alpha}^{\nu} F_{\nu \mu}\right)\left(D^{\mu} \Phi^{\alpha}+v_{\sigma}^{\alpha} F^{\sigma \mu}\right)+\frac{i}{2}(\bar{\lambda} \phi \lambda) \\
&-\frac{1}{2}\left(\left[\phi_{1}, \phi_{2}\right]-\mathscr{L}_{v_{1}} \phi_{2}+\mathscr{L}_{v_{2}} \phi_{1}+v_{1}^{\mu} v_{2}^{\nu} F_{\mu \nu}\right)^{2} \\
&\left.+\frac{i}{2}\left(\bar{\lambda} \gamma_{9}\left(\left[\phi_{1}, \lambda\right]-\mathscr{L}_{v_{1}} \lambda\right)\right)+\frac{1}{2}\left(\bar{\lambda}\left(\left[\phi_{2}, \lambda\right]-\mathscr{L}_{v_{2}} \lambda\right)\right)\right)
\end{aligned}
$$

We recall that the spinor Lie derivative is defined by

$$
\mathscr{L}_{v} \lambda=v^{\mu} D_{\mu} \lambda+\frac{1}{2} D_{\mu} v_{\nu} \gamma^{\mu \nu} \lambda
$$

where $\gamma^{\mu \nu} \equiv \frac{1}{4}\left[\gamma^{\mu}, \gamma^{\nu}\right]$

The action (132) has the following supersymmetry :

$$
\begin{aligned}
\delta A_{\mu}=-i\left(\bar{\epsilon} \gamma_{\mu} \lambda\right) & \\
\delta \phi_{1} & =-\left(\bar{\epsilon} \gamma_{9} \lambda\right)-i\left(\bar{\epsilon} \psi_{1} \lambda\right) \\
\delta \phi_{2}=-(\bar{\epsilon} \lambda)-i\left(\bar{\epsilon} \psi_{2} \lambda\right) & \\
\delta \lambda=\not F \epsilon-i \gamma_{9}\left(\not D \phi_{1}+i_{v_{1}} F\right) \epsilon-i\left(\not D \phi_{2}+i_{v_{2}} F\right) \epsilon & \\
& \quad+\gamma_{9}\left(\left[\phi_{1}, \phi_{2}\right]-\mathscr{L}_{v_{1}} \phi_{2}+\mathscr{L}_{v_{2}} \phi_{2}+i_{v_{2}} i_{v_{1}} F\right) \epsilon
\end{aligned}
$$

In fact, the symmetry holds only if the covariantly constant spinor $\epsilon$ is constant along the flow of the $v_{\alpha}$ 's. Thus $\Omega_{a b}^{\alpha} \equiv \frac{1}{2}\left(d g\left(v^{\alpha}\right)\right)_{a b}$ must define a degenerated matrix $\Omega^{\alpha}$. In eight 
dimensions, a constant spinor only exists on a Joyce manifold, and its Lie derivative can be written as

$$
\mathcal{L}_{v} \varepsilon_{\alpha}=\frac{1}{2} \bigwedge_{\alpha}{ }^{\beta} \varepsilon_{\beta}
$$

which is zero if and only if $\Omega$ is selfdual. Thus the constant spinor remains invariant under the isometries generated by $v_{\alpha}$ 's only if the matrix $\Omega_{\alpha}$ is selfdual. Such vectors verify the third equations in (124) $d \star d g\left(v_{\alpha}\right)=0$. The explicit form of the action (132) for $M \cong \mathbb{R}^{8}$ is displayed in Appendix $\mathrm{C}$.

\subsubsection{The twisted theory for $\Omega$ backgrounds}

The modifications of the supersymmetric theory are formally quite mild when one introduces the $\Omega$ backgrounds. They are completely determined at the purely bosonic level. Thus, all twist operations must remain identical, and one can define the twisted scalar and vector operators $Q$ and $Q_{\mu}$ from Eq.(134). To compute the twisted version of the action (132), we define:

$$
v=v_{1}-v_{2} \quad \bar{v}=\frac{1}{2}\left(v_{1}+v_{2}\right)
$$

We obtain :

$$
\begin{gathered}
I=\int_{M} \operatorname{Tr}\left(-\frac{1}{2} F \star F-\left(d_{A} \Phi-i_{v} F\right) \star\left(d_{A} \bar{\Phi}-i_{\bar{v}} F\right)-4 \chi \star d_{A} \Psi-\Psi \star d_{A} \eta\right. \\
-2 \chi \star\left([\Phi, \chi]+\mathscr{L}_{v} \chi\right)-\frac{1}{2} \eta \star\left([\Phi, \eta]+\mathscr{L}_{v} \eta\right) \\
\left.-\Psi \star\left([\bar{\Phi}, \Psi]+\mathscr{L}_{\bar{v}} \Psi\right)+\frac{1}{2}\left([\Phi, \bar{\Phi}]+\mathscr{L}_{v} \bar{\Phi}-\mathscr{L}_{\bar{v}} \Phi+i_{\bar{v}} i_{v} F\right)^{2}+2 T \star T\right)
\end{gathered}
$$

One can verify that this action is $Q$ exact, up to a topological term and can be written as follows:

$$
\begin{aligned}
I=-\frac{1}{2} \int_{M} \operatorname{Tr}\left(C_{\wedge} F_{\wedge} F\right) & \\
& +Q \int_{M} \operatorname{Tr}\left(-2 \chi \star(F-T)+\Psi \star\left(d_{A} \bar{\Phi}-i_{\bar{v}} F\right)\right. \\
& \left.+\frac{1}{2} \star \eta\left([\Phi, \bar{\Phi}]+\mathscr{L}_{v} \bar{\Phi}-\mathscr{L}_{\bar{v}} \Phi+i_{\bar{v}} i_{v} F\right)\right)
\end{aligned}
$$


We thus recover by twisting the action of the equivariant topological theory $(117)^{8}$, and discover that the scalar and vector topological symmetries defined from the equivariant horizontality conditions correspond to the twisted supersymmetries of the Super YangMills theory on the $\Omega$-background. Notice that the action in the twisted formulation (137) is BRST-exact, and so BRST closed, for all commuting vector fields $v_{\alpha}$, so that the matrices $\Omega_{a b}^{\alpha}$ are no longer required to be self-dual in order to have a BRST-closed action. This can be understood as follows: if we consider the twisted theory for a generic $\Omega$-background (i.e. $\Omega_{a b}^{\alpha}$ generic matrices) and we untwist it, we get a term

$$
\frac{1}{4} \lambda^{\alpha} \bigwedge_{\alpha}^{+\beta} \lambda^{\beta}-\frac{1}{2} \lambda_{\dot{\alpha}}\left(\bar{\Omega}_{\dot{\beta}}^{+\dot{\alpha}}-\bar{\nearrow}_{\dot{\beta}}^{-\dot{\alpha}}\right) \lambda^{\dot{\beta}}
$$

where the plus and the minus stand for the self-dual and anti-self-dual projections with respect to the Cayley four form $C$. The term (139) breaks the rotation invariance from $S O(8)$ to $\operatorname{Spin}(7)$, effectively twisting the theory.

\section{Conclusion}

On manifolds of reducible tangent bundle, the existence of a covariantly constant vector field allows one to extend the horizontality condition. This extension define two nilpotent topological operators, the usual scalar one, and the vector topological operator. These two operators define a closed off-shell algebra, in a globally well-defined way. In order to make contact with known results, we observe that the dimensional reduction on a circle with tangent vector $\kappa$ of this horizontality condition is nothing but the BRST-antiBRST horizontality condition, which also defines two topological charges of a resulting balanced topological theory, as it was defined in [13]. The consistency of the algebra needs the existence of the concept of selfduality or antiselfduality. In eight dimensions, this implies that the manifold is of a Joyce type. The use of the vector symmetry permits one to raise the indetermination of the topological gauge function, and eventually of a topological BRSTQFT action that determines by twist supersymmetry.

The invariance of the action under the vector symmetry is in fact equivalent to the conservation of its Noether current, which turns out to be a BRST-antecedent of the energy momentum tensor. A more conventional construction would be the definition of a BRSTQFT from the last condition, but it would obscure the geometrical interpretation.

\footnotetext{
${ }^{8}$ This hold true, up to a rescaling in (116) which leaves the BRST operator invariant. That is of a factor -2 for $\chi$ and $T$, a factor $\frac{1}{2}$ for $\bar{\Phi}, \eta$ and $\bar{v}$, and a global factor 2 on the action. We must also add the substitution $T \rightarrow T+F^{-}$.
} 
This algebraic construction of topological theory extends to the case of $\Omega$ background. The extended differential is understood as the equivariant differential with respect to the action of an isometry group of the physical space, and the observables of the theory are in the equivariant cohomology of this differential.

Beyond the mathematical interpretation of the fields occurring in BRSTQFT, it is striking that the extended horizontality condition also provides a geometrical construction of a subalgebra of (possibly maximal) supersymmetry which can is closed off-shell and completely determines the action. Thus, it determines the whole supersymmetric algebra in the flat space limit, and the question of having no finite set of auxiliary field for the superPoincaré algebra becomes irrelevant. These results are compatible with dimensional reduction, and apply therefore to different cases of supersymmetry, in other dimensions.

\section{A Algebra of the octonionic 4-form}

We define the two projectors :

$$
\begin{aligned}
P^{-}{ }_{\mu \nu}{ }^{\sigma \rho} & \equiv \frac{1}{4}\left(\delta_{\mu \nu}^{\sigma \rho}-\frac{1}{2} C_{\mu \nu}{ }^{\sigma \rho}\right) \\
P^{+}{ }_{\mu \nu}{ }^{\sigma \rho} & \equiv \frac{3}{4}\left(\delta_{\mu \nu}^{\sigma \rho}+\frac{1}{6} C_{\mu \nu}{ }^{\sigma \rho}\right)
\end{aligned}
$$

and from the formula [34]

$$
C_{\mu \nu \sigma \rho} C^{\kappa \lambda \theta \rho}=6 \delta_{\mu \nu \sigma}^{\kappa \lambda \theta}-9 C_{[\mu \nu}^{[\kappa \lambda} \delta_{\sigma]}^{\theta]}
$$

we can show that

$$
\begin{aligned}
& P^{-}{ }_{\sigma \rho\{\mu}{ }^{\theta} P^{-}{ }_{\nu\} \theta}{ }^{\kappa \lambda}=\frac{1}{8} \delta_{\mu \nu} P^{-}{ }_{\sigma \rho}{ }^{\kappa \lambda} \\
& \left.\left(\delta_{\mu \nu}^{\{\theta \mid \tau}+\frac{1}{2} C_{\mu \nu}{ }^{\{\theta \mid \tau}\right) P_{\tau}^{-\sigma\} \rho \kappa}=-\delta^{\theta[\sigma} P^{-}{ }_{\mu \nu} \rho\right] \kappa \\
& \left(\delta_{\sigma \rho}^{[\mu \mid \theta}+\frac{1}{2} C_{\sigma \rho}^{[\mu \mid \theta}\right) P^{-\kappa \lambda \mid \nu]}{ }_{\theta}=\frac{1}{4} \delta_{[\sigma}^{[\mu} C_{\rho]}{ }^{\nu] \kappa \lambda}+\frac{1}{4} C_{\mu \nu[\sigma}\left[{ }^{[\kappa} \delta_{\rho]}^{\lambda]}\right. \\
& \frac{1}{2} C_{\theta \tau}^{\mu \nu}\left(P_{\sigma \rho}^{-}{ }^{\theta \eta} P^{-\tau}{ }_{\eta \kappa \lambda}\right)=P_{\sigma \rho}^{-}{ }^{[\mu \mid \eta} P^{-\nu]}{ }_{\eta \kappa \lambda} \\
& \frac{1}{2} C_{\theta \tau}^{\mu \nu}\left(P_{\sigma \rho}^{-}{ }^{\theta \eta} P^{+\tau}{ }_{\eta \kappa \lambda}\right)=-3 P_{\sigma \rho}^{-}{ }^{[\mu \mid \eta} P^{+\nu]}{ }_{\eta \kappa \lambda}
\end{aligned}
$$


Actually, the two last equations state that if we consider 2-form as Lie algebra elements, the commutator of two antiselfduals ones gives a selfdual, and the commutator of one antiselfdual and one selfdual gives an antiselfdual. The first and the fourth can be seen from seven dimensional gamma matrix point of view, where $\gamma_{a}$ states for vector (antiselfdual 2-form) and $\gamma_{a b}$ for $\operatorname{Spin}(7)$ Lie algebra element (selfdual 2-form), we see that both equations represent the single formula $\gamma_{a} \gamma_{b}=\delta_{a b}+2 \gamma_{a b}$.

\section{B $\quad \delta_{\mathfrak{k}}$ invariance of the gauge function in $\Omega$ background}

In this appendix, we show that the $\delta$ operator (111) constrains correctly the more general gauge function exactly renormalizable in four dimensions. The gauge function contains, a priori, terms involving the two vectors $v$ and $\bar{v}$. We will assume that the gauge function does not depend of the derivatives of $v$ and $\bar{v}$. These vectors have respectively ghost number 2 and -2 . We can decompose the gauge function of total ghost number -1 into a sum over the terms of field's ghost number $2 i-1$.

$$
\Psi=\sum_{i} \Psi_{i}
$$

In exactly the same way $\delta_{\bar{c}}$ decomposes into $\delta_{-1}+\delta_{0}+\delta_{1}$ and the equation $\delta_{\bar{c}} \Psi=0$ decomposes into

$$
\delta_{1} \Psi_{i-1}+\delta_{0} \Psi_{i}+\delta_{-1} \Psi_{i+1}=0 \quad, \forall i
$$


Since we are interested in the equivariant part of the action, we have not considered the fields $c, \bar{c}$ and $b$. The possible gauge functions of given field's ghost number are

$$
\begin{aligned}
& \Psi_{-1}=\int_{M} \operatorname{Tr}\left(\mathfrak{a}_{-1} i_{v} \chi \star d_{A} \bar{\Phi}+\mathfrak{b}_{-1} \star \eta \mathscr{L}_{v} \bar{\Phi}\right) \\
& \Psi_{0}=\int_{M} \operatorname{Tr}\left(\mathfrak{a}_{0} \chi \star T+\mathfrak{b}_{0} \chi \star F+\mathfrak{c}_{0} \Psi \star d_{A} \bar{\Phi}+\mathfrak{d}_{0} \star \eta[\Phi, \bar{\Phi}]\right. \\
& +\mathfrak{a}_{0}^{\prime} i_{\bar{v}} \chi \star i_{v} T+\overline{\mathfrak{a}}_{0}^{\prime} i_{v} \chi \star i_{\bar{v}} T+\mathfrak{b}_{0}^{\prime} i_{\bar{v}} \chi \star i_{v}(F+\beta \star C F) \\
& +\overline{\mathfrak{b}}_{0}^{\prime} i_{v} \chi \star i_{\bar{v}}(F+\bar{\beta} \star C F)+\mathfrak{c}_{0}^{\prime} \star i_{\bar{v}} \Psi \mathscr{L}_{v} \bar{\Phi}+\overline{\mathfrak{c}}_{0}^{\prime} \star i_{v} \Psi \mathscr{L}_{\bar{v}} \bar{\Phi} \\
& \left.+\mathfrak{d}_{0}^{\prime} \star \eta i_{\bar{v}} i_{v} T+\mathfrak{e}_{0}^{\prime} \star \eta i_{\bar{v}} i_{v}(F+\gamma \star C F)+\mathfrak{f}_{0}^{\prime} \star i_{\bar{v}} i_{v} \chi[\Phi, \bar{\Phi}]\right) \\
& \Psi_{1}=\int_{M} \operatorname{Tr}\left(\mathfrak{a}_{1} i_{\bar{v}} T \star \Psi+\mathfrak{b}_{1} i_{\bar{v}}(F+\alpha \star C F) \star \Psi+\mathfrak{c}_{1} i_{\bar{v}} \chi \star d_{A} \Phi\right. \\
& +\mathfrak{d}_{1} \star \eta \mathscr{L}_{\bar{v}} \Phi+\mathfrak{e}_{1} \star i_{\bar{v}} \Psi[\Phi, \bar{\Phi}] \\
& +|\bar{v}|^{2}\left(\mathfrak{a}_{1}^{\prime} i_{v} T \star \Psi+\mathfrak{b}_{1}^{\prime} i_{v}\left(F+\alpha^{\prime} \star C F\right) \star \Psi\right. \\
& \left.\left.+\mathfrak{c}_{1}^{\prime} i_{v} \chi \star d_{A} \Phi+\mathfrak{d}_{1}^{\prime} \star \eta \mathscr{L}_{v} \Phi+\mathfrak{e}_{1}^{\prime} \star i_{v} \Psi[\Phi, \bar{\Phi}]\right)\right) \\
& \Psi_{2}=\int_{M} \operatorname{Tr}\left(\mathfrak{a}_{2}|\bar{v}|^{2} \Psi \star d_{A} \Phi+\mathfrak{b}_{2} \star i_{\bar{v}} \Psi \mathscr{L}_{\bar{v}} \Phi+|\bar{v}|^{2} \star\left(\mathfrak{c}_{2} i_{\bar{v}} \Psi \mathscr{L}_{v} \Phi+\overline{\mathfrak{c}}_{2} i_{v} \Psi \mathscr{L}_{\bar{v}} \Phi\right)\right)
\end{aligned}
$$

where the parameters could be arbitrary functions of $(v \cdot \bar{v})$. In this computation, we use the fact that $v$ and $\bar{v}$ are two commuting Killing vectors, the fact that $\kappa$ is covariantly constant and, as a matter of fact, we must add to these requirements that these three vectors are linearly independent, that the three 2-form $g(v) \wedge g(\bar{v}), g(\kappa) \wedge g(v)$ and $g(\kappa)_{\wedge} g(\bar{v})$, are not selfdual, as well as the condition that $(\kappa \cdot v)$ is not zero. The easiest way to compute $\delta_{\bar{c}} \Psi=0$ is to begin by the term of higher degree in order to constrain the parameters before to compute the more complex expressions. $\delta_{-1} \Psi_{-1}=0$ does not give any informations, but $\delta_{1} \Psi_{2}=0$ constrains $\mathfrak{a}_{2}$ to be zero. $\delta_{1} \Psi_{1}+\delta_{0} \Psi_{2}=0$ then establishes that $\Psi_{2}$ is null, and that $\mathfrak{b}_{1}$ and $\mathfrak{d}_{1}$ are the only two non zero parameters of $\Psi_{1}$ and must be opposed. Next $\delta_{0} \Psi_{-1}+\delta_{-1} \Psi_{0}=0$ gives $\mathfrak{a}_{-1}, \overline{\mathfrak{c}}_{0}^{\prime}$ and $\mathfrak{f}_{0}^{\prime}$ to be zero and constrains $\mathfrak{b}_{-1}, \mathfrak{c}_{0}$ and $\mathfrak{d}_{0}$ to be equal. The most meaningful equation is $\delta_{1} \Psi_{0}+\delta_{1} \Psi_{0}=0$, it gives $\mathfrak{a}_{0}^{\prime}=\overline{\mathfrak{a}}_{0}^{\prime}=\mathfrak{b}_{0}^{\prime}=\overline{\mathfrak{b}}_{0}^{\prime}=\mathfrak{c}_{0}^{\prime}=\mathfrak{d}_{0}=0$ and constrains all the other parameters. But, let us look at a residual term more closely. After all the coefficients have been constrained 
up to a global factor we leave with the expression

$$
\delta_{1} \Psi_{0}+\delta_{0} \Psi_{1}=\int_{M} \frac{\mathfrak{b}_{0}}{2} g(\kappa)_{\wedge} C_{\wedge} i_{\bar{v}} \operatorname{Tr}\left(F_{\wedge} F\right)
$$

which seems to be non zero at first sight. Since the manifold on which the theory is defined admits a covariantly constant vector field, it must decompose into $\mathbb{R} \times N$ in the simply connected case ${ }^{9}$, as a matter of fact we will be able to annul the term (146) only in this case. The embeddings of $\mathbb{R}$ in $M$ can be put into effect by the flow of $\kappa$, which we remind to be defined by

$$
\frac{d}{d t} \phi_{\kappa, t}(p)=\kappa_{\mid \phi_{\kappa, t}(p)}
$$

This one defines a global function $t$ on $M$, for which the flow equation can be written $d t=g(\kappa)$. Therefore $g(\kappa)$ is $d$-exact and we can use this property to compute that ${ }^{10}$

$$
\int_{M} g(\kappa)_{\wedge} C_{\wedge} i_{\bar{v}} \operatorname{Tr}\left(F_{\wedge} F\right)=\int_{M}\left((\kappa \cdot \bar{v}) C+t \mathcal{L}_{\bar{v}} C\right)_{\wedge} \operatorname{Tr}\left(F_{\wedge} F\right)
$$

In order to this term to be null, $(\kappa \cdot \bar{v})$ must be constant for a gauge fiber bundle of trivial second Chern class, and zero otherwise, and $d g(\bar{v})$ must be selfdual in order for $\mathcal{L}_{\bar{v}}$ to leave $C$ invariant. That is why we have not assumed that $(\kappa \cdot \bar{v})$ was non zero in the calculus. These requirements are a bit strong, because they impose $\bar{v}$ to be zero in four dimensions. Nevertheless the case studied of Nekrasov [24, 25] fall in this restricted class. As a matter of fact $\bar{v}$ does not contribute to topological amplitudes by construction. To finish the computation, note that the last constraint, confirms what has already been given by the others. Therefore we obtain the well constrained gauge function up to a global scale factor

$$
\begin{aligned}
\Psi=\int_{M} \operatorname{Tr}\left(\frac{2}{n} \chi \star T+\chi \star F\right. & +\Psi \star\left(d_{A} \bar{\Phi}-i_{\bar{v}} F\right) \\
& \left.+\star \eta\left([\Phi, \bar{\Phi}]+\mathscr{L}_{v} \bar{\Phi}-\mathscr{L}_{\bar{v}} \Phi+i_{\bar{v}} i_{v} F\right)\right)
\end{aligned}
$$

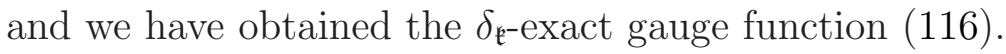

\footnotetext{
${ }^{9}$ See page 16 .

${ }^{10}$ Note that in order to the integration by part to work, $F$ must converges as quickly as necessary where $t \rightarrow \infty$ in such way that the $t$ factor could be compensated.
} 


\section{C $\Omega$ background in Euclidean space}

In the case of a flat space, we can write explicitly the form of the Killing vectors as generators of $\mathfrak{s o}(n)$ elements. We give here the explicit form of the supersymmetric action in the eight dimensional case. We use the scalar $\Phi$ and $\bar{\Phi}$ used in the twisted version, as well as the matrix $\Omega_{\mu \nu} \equiv \frac{1}{2}(d g(v))_{\mu \nu}$ and $\bar{\Omega}_{\mu \nu} \equiv \frac{1}{2}(d g(\bar{v}))_{\mu \nu}$. With these definitions the $\Omega$ background action can be expended as follows :

$$
\begin{aligned}
& S=S_{0}+\bar{\Omega}^{\mu}{ }_{\nu} \bar{S}_{1 \mu}{ }^{\nu}+\Omega^{\mu}{ }_{\nu} S_{1 \mu}{ }^{\nu} \\
& +\Omega^{\mu}{ }_{\sigma} \Omega^{\nu}{ }_{\rho} S_{2 \mu \nu}{ }^{\sigma \rho}+\Omega^{\mu}{ }_{\sigma} \bar{\Omega}_{\rho}^{\nu} S_{2 \mu \nu}^{(1,1) \sigma \rho}+\bar{\Omega}^{\mu}{ }_{\sigma} \bar{\Omega}_{\rho}^{\nu} \bar{S}_{2 \mu \nu}{ }^{\sigma \rho} \\
& +\bar{\Omega}^{\mu}{ }_{\sigma} \bar{\Omega}^{\nu}{ }_{\rho} \Omega^{\kappa}{ }_{\lambda} \bar{S}_{3 \mu \nu \kappa}{ }^{\sigma \rho \lambda}+\Omega^{\mu}{ }_{\sigma} \Omega^{\nu}{ }_{\rho} \bar{\Omega}^{\kappa}{ }_{\lambda} S_{3 \mu \nu \kappa}{ }^{\sigma \rho \lambda} \\
& +\Omega^{\mu}{ }_{\sigma} \bar{\Omega}_{\rho}^{\nu} \Omega^{\kappa}{ }_{\theta} \bar{\Omega}^{\lambda}{ }_{\tau} S_{4 \mu \nu \kappa \lambda}{ }^{\sigma \rho \theta \tau}
\end{aligned}
$$

with the definitions

$$
\begin{aligned}
S_{0} & \equiv \int_{M} d^{8} x \operatorname{Tr}\left(-\frac{1}{4} F_{\mu \nu} F^{\mu \nu}+D_{\mu} \bar{\Phi} D^{\mu} \Phi-\frac{i}{2} \lambda^{\alpha} D_{\alpha \dot{\alpha}} \lambda^{\dot{\alpha}}-\frac{i}{2} \lambda_{\dot{\alpha}} D^{\dot{\alpha} \alpha} \lambda_{\alpha}\right. \\
& \left.+\frac{1}{2}[\Phi, \bar{\Phi}]^{2}+\lambda_{\dot{\alpha}}\left[\bar{\Phi}, \lambda^{\dot{\alpha}}\right]-\frac{1}{2} \lambda^{\alpha}\left[\Phi, \lambda_{\alpha}\right]\right) \\
\bar{S}_{1 \mu}{ }^{\nu} & \equiv \int_{M} d^{8} x\left(x^{\nu} \operatorname{Tr}\left(F_{\mu \sigma} D^{\sigma} \Phi+[\Phi, \bar{\Phi}] D_{\mu} \Phi-\lambda_{\dot{\alpha}} D_{\mu} \lambda^{\dot{\alpha}}\right)+\frac{1}{2} \operatorname{Tr}\left(\lambda_{\dot{\alpha}} \gamma_{\mu}{ }^{\nu \dot{\alpha}}{ }_{\dot{\beta}} \lambda^{\dot{\beta}}\right)\right) \\
S_{1 \mu}{ }^{\nu} & \equiv \int_{M} d^{8} x\left(x^{\nu} \operatorname{Tr}\left(F_{\mu \sigma} D^{\sigma} \bar{\Phi}-[\Phi, \bar{\Phi}] D_{\mu} \bar{\Phi}+\frac{1}{2} \lambda^{\alpha} D_{\mu} \lambda_{\alpha}\right)-\frac{1}{4} \operatorname{Tr}\left(\lambda^{\alpha} \gamma_{\mu}{ }^{\nu}{ }_{\alpha}{ }^{\beta} \lambda_{\beta}\right)\right) \\
S_{2 \mu \nu}{ }^{\sigma \rho} & \equiv \frac{1}{2} \int_{M} d^{8} x x^{\sigma} x^{\rho} \operatorname{Tr}\left(D_{\mu} \bar{\Phi} D_{\nu} \bar{\Phi}\right) \\
S_{2 \mu \nu}^{(1,1) \sigma \rho} & \equiv \int_{M} d^{8} x x^{\sigma} x^{\rho} \operatorname{Tr}\left(F_{\mu}{ }^{\kappa} F_{\nu \kappa}+F_{\mu \nu}[\Phi, \bar{\Phi}]-D_{\mu} \bar{\Phi} D_{\nu} \Phi\right) \\
\bar{S}_{2 \mu \nu}{ }^{\sigma \rho} & \equiv \frac{1}{2} \int_{M} d^{8} x x^{\sigma} x^{\rho} \operatorname{Tr}\left(D_{\mu} \Phi D_{\nu} \Phi\right) \\
\bar{S}_{3 \mu \nu \kappa}{ }^{\sigma \rho \lambda} & \equiv \int_{M} d^{8} x x^{\sigma} x^{\rho} x^{\lambda} \operatorname{Tr}\left(F_{\kappa \mu} D_{\nu} \Phi\right) \\
S_{3 \mu \nu \kappa}{ }^{\sigma \rho \lambda} & \equiv \int_{M} d^{8} x x^{\sigma} x^{\rho} x^{\lambda} \operatorname{Tr}\left(F_{\kappa \mu} D_{\nu} \bar{\Phi}\right) \\
S_{4 \mu \nu \kappa \lambda}{ }^{\sigma \rho \theta \tau} & \equiv \frac{1}{2} \int_{M} d^{8} x x^{\sigma} x^{\rho} x^{\theta} x^{\tau} \operatorname{Tr}\left(F_{\mu \nu} F_{\kappa \lambda}\right)
\end{aligned}
$$




\section{Acknowledgments}

This work was partially supported under the contract ANR(CNRS-USAR) no. 05-BLAN-0079-01.

\section{References}

[1] L. Baulieu, H. Kanno and I. M. Singer, "Special quantum field theories in eight and other dimensions," Commun. Math. Phys. 194 (1998) 149 [arXiv:hep-th/9704167].

[2] S. K. Donaldson and R. P. Thomas, "Gauge theory in higher dimensions", in "The geometric universe: Science, geometry, and the work of Roger Penrose. Proceedings, Symposium, Geometric Issues in the Foundations of Science, Oxford, UK, June 25-29, 1996,"

[3] B. S. Acharya, M. O'Loughlin and B. Spence, "Higher-dimensional analogues of Donaldson-Witten theory," Nucl. Phys. B 503 (1997) 657 [arXiv:hep-th/9705138].

[4] D. Birmingham, M. Rakowski and G. Thompson, "Renormalization Of Topological Field Theory," Nucl. Phys. B 329, 83 (1990).

[5] M. Blau and G. Thompson, "Euclidean SYM theories by time reduction and special holonomy manifolds," Phys. Lett. B 415, 242 (1997) [arXiv:hep-th/9706225].

[6] L. Baulieu, "Gravitational topological quantum field theory versus $\mathcal{N}=2 D=8$ supergravity," JHEP 0404, 044 (2004) [arXiv:hep-th/0304221].

[7] O. Piguet, "On the role of vector supersymmetry in topological field theory," [arXiv:hep-th/9502033].

[8] F. Gieres, J. Grimstrup, T. Pisar and M. Schweda, "Vector supersymmetry in topological field theories," JHEP 0006, 018 (2000) [arXiv:hep-th/0002167].

[9] F. Fucito, A. Tanzini, L. C. Q. Vilar, O. S. Ventura, C. A. G. Sasaki and S. P. Sorella, "Algebraic renormalization: Perturbative twisted considerations on topological Yang-Mills theory and on $\mathcal{N}=2$ supersymmetric gauge theories," [arXiv:hep-th/9707209]; A. Tanzini, O. S. Ventura, L. C. Q. Vilar and 
S. P. Sorella, "BRST cohomology of $\mathcal{N}=2$ super-Yang-Mills theory in four dimensions," J. Phys. G 26, 1117 (2000) [arXiv:hep-th/9811191]; A. Blasi, V. E. R. Lemes, N. Maggiore, S. P. Sorella, A. Tanzini, O. S. Ventura and L. C. Q. Vilar, "Perturbative beta function of $\mathcal{N}=2$ super Yang-Mills theories," JHEP 0005, 039 (2000) [arXiv:hep-th/0004048]; V. E. R. Lemes, M. S. Sarandy, S. P. Sorella, A. Tanzini and O. S. Ventura, "The action of $\mathcal{N}=4$ super Yang-Mills from a chiral primary operator," JHEP 0101, 016 (2001) [arXiv:hep-th/0011001]; V. E. R. Lemes, N. Maggiore, M. S. Sarandy, S. P. Sorella, A. Tanzini and O. S. Ventura, "Nonrenormalization theorems for $\mathcal{N}=2$ super Yang-Mills," [arXiv:hep-th/0012197]; N. Maggiore and A. Tanzini, "Protected operators in $\mathcal{N}=2$, 4 supersymmetric theories," Nucl. Phys. B 613, 34 (2001) [arXiv:hep-th/0105005]; R. Flume, "Some remarks on $\mathcal{N}=2$ extended supersymmetric Yang-Mills theories and Seiberg-Witten duality," [arXiv:hep-th/9702192]; K. Ulker, "N $\mathcal{N}=2$ super Yang Mills action and BRST cohomology," Mod. Phys. Lett. A 19 (2004) 713 [arXiv:hep-th/0307279]; K. Ulker, "N $=2$ super Yang Mills action as a BRST exact term, topological Yang Mills and instantons," Phys. Rev. D 68, 085005 (2003) [arXiv:hep-th/0304154].

[10] L. Baulieu and J. Thierry-Mieg, "Algebraic Structure Of Quantum Gravity And The Classification Of The Gravitational Anomalies," Phys. Lett. B 145, 53 (1984).

[11] L. Baulieu and M. Bellon, "A Simple Algebraic Construction Of The Symmetries Of Supergravity," Phys. Lett. B 161, 96 (1985).

[12] F. Langouche, T. Schucker and R. Stora, "Gravitational Anomalies Of The AdlerBardeen Type," Phys. Lett. B 145, 342 (1984).

[13] R. Dijkgraaf and G. W. Moore, "Balanced topological field theories," Commun. Math. Phys. 185, 411 (1997) [arXiv:hep-th/9608169].

[14] G. De Rham, Sur la réducibilité d'un espace de Riemann, Commentarii Mathematici Helvetici 26, 328 (1952).

[15] L. Baulieu and C. Laroche, "On generalized self-duality equations towards supersymmetric quantum field theories of forms," Mod. Phys. Lett. A 13, 1115 (1998) [arXiv:hep-th/9801014].

[16] S. Ouvry, R. Stora and P. van Baal, "On The Algebraic Characterization Of Witten's Topological Yang-Mills Theory," Phys. Lett. B 220, 159 (1989). 
[17] L. Baulieu, P. A. Grassi and D. Zwanziger, "Gauge and topological symmetries in the bulk quantization of gauge theories," Nucl. Phys. B 597, 583 (2001) [arXiv:hep-th/0006036].

[18] S. Weinberg, "Gravitation and cosmology", John Wiley 1972, section 12.5.

[19] S. Cordes, G. W. Moore and S. Ramgoolam, "Lectures on 2-d Yang-Mills theory, equivariant cohomology and topological field theories," Nucl. Phys. Proc. Suppl. 41, 184 (1995) [arXiv:hep-th/9411210].

[20] D. Mulsch and B. Geyer, "Cohomological extension of Spin(7)-invariant super Yang-Mills theory in eight dimensions," Nucl. Phys. B 684, 351 (2004) [arXiv:hep-th/0310275].

[21] Dominic D. Joyce, "Compact manifolds with special holonomy", Oxford university press 2000.

[22] N. Nekrasov and A. Okounkov, "Seiberg-Witten theory and random partitions," [arXiv:hep-th/0306238].

[23] P. van Nieuwenhuizen and A. Waldron, "A continuous Wick rotation for spinor fields and supersymmetry in Euclidean space," [arXiv:hep-th/9611043].

[24] N. A. Nekrasov, "Seiberg-Witten prepotential from instanton counting," Adv. Theor. Math. Phys. 7, 831 (2004) [arXiv:hep-th/0206161].

[25] T. Eguchi and H. Kanno, "Topological strings and Nekrasov's formulas," JHEP 0312, 006 (2003) [arXiv:hep-th/0310235].

[26] L. Baulieu and I. M. Singer, "Topological Yang-Mills Symmetry," Nucl. Phys. Proc. Suppl. 5B, 12 (1988).

[27] D. Bellisai, F. Fucito, A. Tanzini and G. Travaglini, "Instanton calculus, topological field theories and $\mathcal{N}=2$ super Yang-Mills theories," JHEP 0007 (2000) 017 [arXiv:hep-th/0003272]; R. Flume, R. Poghossian and H. Storch, "The SeibergWitten prepotential and the Euler class of the reduced moduli space of instantons," Mod. Phys. Lett. A 17 (2002) 327 [arXiv:hep-th/0112211].

[28] N. Berline, E. Getzler and M. Vergne, "Heat kernels and Dirac operators", SpringerVerlag Berlin Heidelberg 1992. 
[29] U. Bruzzo, F. Fucito, J. F. Morales and A. Tanzini, "Multi-instanton calculus and equivariant cohomology," JHEP 0305, 054 (2003) [arXiv:hep-th/0211108].

[30] N. A. Nekrasov, "Seiberg-Witten prepotential from instanton counting," Adv. Theor. Math. Phys. 7, 831 (2004) [arXiv:hep-th/0206161].

[31] R. Flume and R. Poghossian, "An algorithm for the microscopic evaluation of the coefficients of the Seiberg-Witten prepotential," Int. J. Mod. Phys. A 18, 2541 (2003) [arXiv:hep-th/0208176].

[32] U. Bruzzo and F. Fucito, "Superlocalization formulas and supersymmetric YangMills theories," Nucl. Phys. B 678 (2004) 638 [arXiv:math-ph/0310036].

[33] R. Flume, F. Fucito, J. F. Morales and R. Poghossian, "Matone's relation in the presence of gravitational couplings," JHEP 0404 (2004) 008 [arXiv: hep-th/0403057].

[34] A. Bilal, J. P. Derendinger and K. Sfetsos, "(Weak) $G_{2}$ holonomy from self-duality, flux and supersymmetry," Nucl. Phys. B 628, 112 (2002) [arXiv:hep-th/0111274]. 